\title{
Extended superconformal higher-spin gauge theories in four dimensions
}

\section{Sergei M. Kuzenko and Emmanouil S. N. Raptakis}

Department of Physics M013, The University of Western Australia 35 Stirling Highway, Perth W.A. 6009, Australia

E-mail: sergei.kuzenko@uwa.edu.au, emmanouil.raptakis@research.uwa.edu. au

ABSTRACT: Using the off-shell formulation for $\mathcal{N}=2$ conformal supergravity in four dimensions, we describe superconformal higher-spin multiplets of conserved currents in a curved background and present their associated unconstrained gauge prepotentials. The latter are used to construct locally superconformal chiral actions, which are demonstrated to be gauge invariant in arbitrary conformally flat backgrounds. The main $\mathcal{N}=2$ results are then generalised to the $\mathcal{N}$-extended case. We also present the gauge-invariant field strengths for on-shell massless higher-spin $\mathcal{N}=2$ supermultiplets in anti-de Sitter space. These field strengths prove to furnish representations of the $\mathcal{N}=2$ superconformal group.

Keywords: Extended Supersymmetry, Supergravity Models, Superspaces

ArXiv EPrint: 2104.10416 


\section{Contents}

1 Introduction 1

2 Conformal higher-spin supercurrents $\quad 2$

$2.1 \mathcal{N}=2$ conserved current supermultiplets 3

2.2 Reduction to $\mathcal{N}=1$ superspace 4

$3 \mathcal{N}=2$ superconformal higher-spin gauge models 5

3.1 Superconformal models for the $\Upsilon_{\alpha(m) \dot{\alpha}(n)}$ prepotentials 6

3.2 Superconformal models for the $\Upsilon_{\alpha(m)}$ prepotentials 7

3.3 Linearised $\mathcal{N}=2$ conformal supergravity 8

3.4 Reduction to $\mathcal{N}=1$ superspace $\quad 9$

$4 \mathcal{N}>2$ superconformal higher-spin gauge theories $\quad 10$

$4.1 \mathcal{N}>2$ conformal superspace: conformally flat case 11

$\begin{array}{lll}4.2 & \text { Conformal supercurrent multiplets and gauge prepotentials } & 12\end{array}$

$\begin{array}{lll}4.3 & \text { Superconformal gauge-invariant models } & 13\end{array}$

5 Conclusion $\quad 14$

$5.1 \mathcal{N}=2$ superconformal higher-spin gauge theories in $\mathrm{AdS}_{4} \quad 14$

$5.2 \mathcal{N}=2$ superconformal higher-spin models as induced actions $\quad 16$

$\begin{array}{ll}5.3 \mathcal{N}=2 \text { superconformal vector and gravitino multiplets } & 17\end{array}$

5.4 Massless higher-spin $\mathcal{N}=2$ supermultiplets in $\mathrm{AdS}_{4} \quad 18$

A $\mathcal{N}=2$ conformal superspace in four dimensions 19

\section{Introduction}

In four dimensions, off-shell $\mathcal{N}=1$ superconformal higher-spin prepotentials were briefly discussed by Howe, Stelle and Townsend in the framework of supercurrent multiplets in 1981 [1], a few years before Fradkin and Tseytlin [2] constructed the free conformal higherspin actions in Minkowski space. It was only in the last few years that these prepotentials and more general off-shell gauge supermultiplets were finally used to construct free $\mathcal{N}=1$ superconformal higher-spin gauge theories in Minkowski space [3] and arbitrary conformally flat and Bach flat backgrounds [4-7]. Parallel studies in three dimensions (3D) to describe superconformal higher-spin multiplets and the corresponding Chern-Simons actions were conducted [8-13]. These 3D and 4D off-shell constructions open the possibility to develop a manifestly supersymmetric setting for superconformal higher-spin gauge theories which 
were considered for the first time by Fradkin and Linetsky [14, 15] in the component approach. ${ }^{1}$

One of the goals of this paper is to extend some of the results of [3-6] to the $4 \mathrm{D}$ $\mathcal{N}=2$ superconformal case. Using the $\mathcal{N}=2$ conformal superspace approach [19], we will propose superconformal higher-spin multiplets of conserved currents and their associated unconstrained gauge prepotentials. The corresponding superconformal gauge-invariant actions will be constructed in arbitrary conformally flat backgrounds. We will also generalise the main $\mathcal{N}=2$ results to the $\mathcal{N}$-extended case.

Our approach to determine the structure of superconformal higher-spin gauge prepotentials is to use the method of supercurrent multiplets $[1,20,21]$. It is well known that the multiplets of (conformal) currents furnish off-shell representations of (conformal) supersymmetry. Once a conformal higher-spin supercurrent $J$ is known, the associated gauge prepotential $\Upsilon$ is determined via the Noether coupling

$$
S_{\mathrm{NC}}=\int \mathrm{d}^{4} x \mathrm{~d}^{4} \theta \mathrm{d}^{4} \bar{\theta} E \Upsilon \cdot J
$$

This procedure is concisely described by Bergshoeff et al. [21]: "One first assigns a field to each component of the current multiplet, and forms a generalized inner product of field and current components."

In this work we make use of $\mathcal{N}=2$ conformal superspace [19], which is an ultimate formulation for $\mathcal{N}=2$ conformal supergravity in the sense that any different off-shell formulation is either equivalent to it or is obtained from it by partially fixing the gauge freedom. In particular, $\mathrm{U}(2)$ superspace [22] and SU(2) superspace [23] can be derived from conformal superspace by imposing partial gauge fixing conditions. ${ }^{2}$ At the component level, $\mathcal{N}=2$ conformal superspace reduces to the $\mathcal{N}=2$ superconformal tensor calculus [21, $25,26]$. The recently discovered supertwistor formulation for $\mathcal{N}=2$ supergravity [27] is believed to be equivalent to conformal superspace, however the technical details are yet to be worked out.

This paper is organised as follows. In section 2 we determine all $\mathcal{N}=2$ conformal supercurrents. This in turn allows us to compute their dual gauge prepotentials and corresponding gauge invariant actions in conformally flat backgrounds in section 3 . Our key $\mathcal{N}=2$ results are generalised to the $\mathcal{N}$-extended case in section 4 . Concluding comments and implications of the obtained results are given in section 5 . The main body of this paper is accompanied by a technical appendix reviewing the relevant aspects of $\mathcal{N}=2$ conformal superspace.

\section{Conformal higher-spin supercurrents}

The objective of this section is to identify all possible $\mathcal{N}=2$ conformal higher-spin supercurrents $J$ in a curved background, which will in turn elucidate the structure of their dual

\footnotetext{
${ }^{1}$ The Fradkin-Linetsky formulation [14, 15] may be viewed as a natural extension of the Fradkin-Vasiliev approach to massless higher-spin gauge theories [16-18].

${ }^{2}$ The relationship between the $\mathrm{U}(2)$ and $\mathrm{SU}(2)$ superspaces is described in [24].
} 
gauge prepotentials $\Upsilon$. The technical details regarding $\mathcal{N}=2$ conformal superspace are given in the appendix.

\section{$2.1 \mathcal{N}=2$ conserved current supermultiplets}

Let $m$ and $n$ be positive integers. A primary tensor superfield $J^{\alpha(m) \dot{\alpha}(n)}$ defined on the background superspace will be called a conformal supercurrent if it obeys

$$
\begin{array}{lll}
\nabla_{\beta}^{i} J^{\beta \alpha(m-1) \dot{\alpha}(n)}=0 & \Longrightarrow & \nabla^{i j} J^{\alpha(m) \dot{\alpha}(n)}=0, \\
\bar{\nabla}_{\dot{\beta}}^{i} J^{\alpha(m) \dot{\beta} \dot{\alpha}(n-1)}=0 & \Longrightarrow & \bar{\nabla}^{i j} J^{\alpha(m) \dot{\alpha}(n)}=0,
\end{array}
$$

where we have denoted

$$
\nabla^{i j}=\nabla^{\alpha(i} \nabla_{\alpha}^{j)}, \quad \bar{\nabla}^{i j}=\bar{\nabla}_{\dot{\alpha}}^{(i} \bar{\nabla}^{\dot{\alpha} j)} .
$$

These constraints uniquely fix the superconformal properties of $J^{\alpha(m) \dot{\alpha}(n)}$

$$
\mathbb{D} J^{\alpha(m) \dot{\alpha}(n)}=\frac{1}{2}(m+n+4) J^{\alpha(m) \dot{\alpha}(n)}, \quad Y J^{\alpha(m) \dot{\alpha}(n)}=-(m-n) J^{\alpha(m) \dot{\alpha}(n)} .
$$

For $m=n=s, J^{\alpha(s) \dot{\alpha}(s)}$ is invariant under $\mathrm{U}(1)_{R}$ transformations and thus we take it to be real. This special case was first described in Minkowski superspace in [1].

When $n=0$, the constraints (2.1) should be replaced with

$$
\begin{aligned}
\nabla_{\beta}^{i} J^{\beta \alpha(m-1)} & =0 \quad \Longrightarrow \quad \nabla^{i j} J^{\alpha(m)}=0, \\
\bar{\nabla}^{i j} J^{\alpha(m)} & =0 .
\end{aligned}
$$

Consistency of (2.4) with the superconformal algebra implies:

$$
\mathbb{D} J^{\alpha(m)}=\frac{1}{2}(m+4) J^{\alpha(m)}, \quad Y J^{\alpha(m)}=-m J^{\alpha(m)} .
$$

Finally, for the special case $m=0$, we replace (2.4) with

$$
\nabla^{i j} J=0, \quad \bar{\nabla}^{i j} J=0 .
$$

These imply:

$$
\mathbb{D} J=2 J, \quad Y J=0 .
$$

Taking $J$ to be real, it is clear that it corresponds to the conformal supercurrent [1, 28, 29].

Analogous to the $\mathcal{N}=1$ case [5], conformal Killing tensors may be utilised to construct new conserved conformal currents from existing ones. The former are primary tensor superfields $\zeta_{\alpha(p) \dot{\alpha}(q)}, p, q \geq 0$, satisfying

$$
\nabla_{\left(\alpha_{1}\right.}^{i} \zeta_{\left.\alpha_{2} \ldots \alpha_{p+1}\right) \dot{\alpha}(q)}=0, \quad \bar{\nabla}_{\left(\dot{\alpha}_{1}\right.}^{i} \zeta_{\left.\alpha(p) \dot{\alpha}_{2} \ldots \dot{\alpha}_{q+1}\right)}=0 .
$$

In particular, given a conformal supercurrent $J^{\alpha(m) \dot{\alpha}(n)}$ and a conformal Killing tensor $\zeta_{\alpha(p) \dot{\alpha}(q)}$, with $m \geq p$ and $n \geq q$, it may be shown that

$$
\mathfrak{J}^{\alpha(m-p) \dot{\alpha}(n-q)}=J^{\alpha(m-p) \beta(p) \dot{\alpha}(n-q) \dot{\beta}(q)} \zeta_{\beta(p) \dot{\beta}(q)},
$$

is also a conformal supercurrent. The special cases $p=q$ and $p=q=2$ were introduced in [30] and [31], respectively. In a forthcoming work, we will further explore their properties, in particular, their role in the study of higher symmetries [32], see also [33]. 


\subsection{Reduction to $\mathcal{N}=1$ superspace}

To conclude our discussion of the conformal higher-spin supercurrents, it is instructive to comment on the $\mathcal{N}=1$ supermultiplets contained within the conformal supercurrents described above. This analysis requires us to turn off the super-Weyl tensor, that is:

$$
W_{\alpha \beta}=0 .
$$

Let $\nabla_{\alpha}, \bar{\nabla}^{\dot{\alpha}}$ and $\nabla_{\alpha \dot{\alpha}}=\frac{i}{2}\left\{\nabla_{\alpha}, \bar{\nabla}_{\dot{\alpha}}\right\}$ be the covariant derivatives of $\mathcal{N}=1$ conformal superspace [34]. We will define them using the $\mathcal{N}=2$ covariant derivatives (A.1) as follows: $\nabla_{\alpha} \mathfrak{U}=\nabla \frac{1}{\alpha} U \mid$ and $\bar{\nabla}^{\dot{\alpha}} \mathfrak{U}=\bar{\nabla}_{1}^{\dot{\alpha}} U \mid$. Here $U$ is an $\mathcal{N}=2$ superfield, and $\mathfrak{U} \equiv U \mid:=$ $\left.U\right|_{\theta_{\underline{2}}^{\alpha}=\bar{\theta}_{\dot{\alpha}}^{2}=0}$ is its $\mathcal{N}=1$ projection. We will say that $\mathfrak{U}$ is primary if $\mathbf{S}^{\alpha} \mathfrak{U} \equiv S_{\underline{1}}^{\alpha} U \mid=0$ and $\overline{\mathbf{S}}_{\dot{\alpha}} \mathfrak{U} \equiv \bar{S}_{\dot{\alpha}}^{1} U \mid=0$. Using these definitions, one may derive the important identities

$$
\left\{\mathbf{S}^{\alpha}, \nabla_{\beta}\right\}=\delta_{\beta}^{\alpha}(2 \mathbb{D}-3 \mathbb{Y})-4 M_{\beta}^{\alpha}, \quad\left\{\overline{\mathbf{S}}_{\dot{\alpha}}, \bar{\nabla}^{\dot{\beta}}\right\}=\delta_{\dot{\alpha}}^{\dot{\beta}}(2 \mathbb{D}+3 \mathbb{Y})+4 \bar{M}_{\dot{\alpha}}{ }^{\dot{\beta}},
$$

where $\mathbb{Y}$ is the $\mathrm{U}(1)_{R}$ generator. It is related to the $\mathcal{N}=2$ R-symmetry generators via

$$
\mathbb{Y}=\frac{1}{3} Y-\frac{4}{3} J_{\underline{1}} \underline{1}
$$

We now review the key properties of $\mathcal{N}=1$ conformal current supermultiplets (see [35] and [5] for a complete discussion in flat and curved backgrounds, respectively). A primary tensor superfield $\mathcal{J}^{\alpha(m) \dot{\alpha}(n)}$, with $m, n \geq 1$, obeying the constraints

$$
\begin{array}{rll}
\nabla_{\beta} \mathcal{J}^{\beta \alpha(m-1) \dot{\alpha}(n)}=0 & \Longrightarrow & \nabla^{2} \mathcal{J}^{\alpha(m) \dot{\alpha}(n)}=0, \\
\bar{\nabla}_{\dot{\beta}} \mathcal{J}^{\alpha(m) \dot{\beta} \dot{\alpha}(n-1)}=0 & \Longrightarrow & \bar{\nabla}^{2} \mathcal{J}^{\alpha(m) \dot{\alpha}(n)}=0,
\end{array}
$$

is a conformal supercurrent. The $m=n=1$ case corresponds to the ordinary conformal supercurrent [36]. For case $m>n=0$, the appropriate constraints are

$$
\begin{aligned}
\boldsymbol{\nabla}_{\beta} \mathcal{J}^{\beta \alpha(m-1)} & =0 \quad \Longrightarrow \quad \nabla^{2} \mathcal{J}^{\alpha(m)}=0, \\
\bar{\nabla}^{2} \mathcal{J}^{\alpha(m)} & =0
\end{aligned}
$$

The $m=1$ case was first considered in [29], where it was shown that the spinor supercurrent $J^{\alpha}$ naturally originates from the reduction of the conformal $\mathcal{N}=2$ supercurrent [28] to $\mathcal{N}=1$ superspace. Finally, when $m=0$ the supercurrent satisfies

$$
\nabla^{2} \mathcal{J}=0, \quad \bar{\nabla}^{2} \mathcal{J}=0
$$

This is the flavour current supermultiplet [37].

Returning to the reduction procedure, we first consider the $\mathcal{N}=2$ supercurrent $J^{\alpha(m) \dot{\alpha}(n)}(2.1)$. It contains four independent $\mathcal{N}=1$ conserved current supermultiplets 


$$
\begin{aligned}
& j^{\alpha(m) \dot{\alpha}(n)}=J^{\alpha(m) \dot{\alpha}(n)} \mid, \\
& j^{\alpha(m+1) \dot{\alpha}(n)}=\nabla^{\left(\alpha_{1} \underline{2}\right.} J^{\left.\alpha_{2} \ldots \alpha_{m+1}\right) \dot{\alpha}(n)} \mid, \\
& j^{\alpha(m) \dot{\alpha}(n+1)}=\bar{\nabla}_{\underline{2}}^{\left(\dot{\alpha}_{1}\right.} J^{\left.\alpha(m) \dot{\alpha}_{2} \ldots \dot{\alpha}_{n+1}\right)} \mid, \\
& j^{\alpha(m+1) \dot{\alpha}(n+1)}=\frac{1}{2}\left[\nabla^{\left(\alpha_{1} \underline{2}\right.}, \bar{\nabla}_{\underline{2}}^{\left(\dot{\alpha}_{1}\right.}\right] J^{\left.\left.\alpha_{2} \ldots \alpha_{m+1}\right) \dot{\alpha}_{2} \ldots \dot{\alpha}_{n+1}\right)} \mid \\
& -\frac{1}{2(m+n+3)}\left[\boldsymbol{\nabla}^{\left(\alpha_{1}\right.}, \bar{\nabla}^{\left(\dot{\alpha}_{1}\right.}\right] j^{\left.\left.\alpha_{2} \ldots \alpha_{m+1}\right) \dot{\alpha}_{2} \ldots \dot{\alpha}_{n+1}\right)} \\
& -\frac{\mathrm{i}(m-n)}{m+n+3} \boldsymbol{\nabla}^{\left(\alpha _ { 1 } \left(\dot{\alpha}_{1}\right.\right.} j^{\left.\left.\alpha_{2} \ldots \alpha_{m+1}\right) \dot{\alpha}_{2} \ldots \dot{\alpha}_{n+1}\right)} \text {. }
\end{aligned}
$$

Similarly, $J^{\alpha(m)}(2.4)$ is composed of four $\mathcal{N}=1$ supercurrents

$$
\begin{aligned}
j^{\alpha(m)}= & J^{\alpha(m)} \mid \\
j^{\alpha(m+1)}= & \nabla^{\left(\alpha_{1} \underline{2}\right.} J^{\left.\alpha_{2} \ldots \alpha_{m+1}\right)} \mid \\
j^{\alpha(m) \dot{\alpha}}= & \bar{\nabla}_{\underline{2}}^{\dot{\alpha}} J^{\alpha(m)} \mid \\
j^{\alpha(m+1) \dot{\alpha}}= & \frac{1}{2}\left[\nabla^{\left(\alpha_{1} \underline{2}\right.}, \bar{\nabla}_{\underline{2}}^{\dot{\alpha}}\right] J^{\left.\alpha_{2} \ldots \alpha_{m+1}\right)} \mid-\frac{1}{2(m+3)}\left[\nabla^{\left(\alpha_{1}\right.}, \bar{\nabla}^{\dot{\alpha}}\right] j^{\left.\alpha_{2} \ldots \alpha_{m+1}\right)} \\
& -\frac{\mathrm{i} m}{m+3} \nabla^{\left(\alpha_{1} \dot{\alpha}\right.} j^{\left.\alpha_{2} \ldots \alpha_{m+1}\right)} .
\end{aligned}
$$

Finally, upon reduction of $J(2.6)$ we obtain three $\mathcal{N}=1$ current multiplets [29]

$$
\begin{aligned}
j & =J \mid \\
j^{\alpha} & =\nabla^{\alpha \underline{2}} J \mid, \\
j^{\alpha \dot{\alpha}} & =\frac{1}{2}\left[\nabla^{\alpha \underline{2}}, \bar{\nabla}_{\underline{2}}^{\dot{\alpha}}\right] J \mid-\frac{1}{6}\left[\boldsymbol{\nabla}^{\alpha}, \bar{\nabla}^{\dot{\alpha}}\right] j .
\end{aligned}
$$

For further details regarding their dual gauge prepotentials and the superconformal field theories they induce, we refer the reader to [3-6]. See also [7] for their higher-depth generalisations.

\section{$3 \mathcal{N}=2$ superconformal higher-spin gauge models}

In the previous section, we described the $\mathcal{N}=2$ conformal higher-spin supercurrents. Here, by requiring that the Noether coupling (1.1) is superconformal and gauge-invariant, we will identify their dual gauge prepotentials.

The first family of supercurrents take the form $J^{\alpha(m) \dot{\alpha}(n)}, m, n \geq 1$, and are subject to (2.1). It then follows that their duals, $\Upsilon_{\alpha(m) \alpha(n)}$, are defined modulo gauge transformations

$$
\delta_{\zeta, \lambda} \Upsilon_{\alpha(m) \dot{\alpha}(n)}=\nabla_{\left(\alpha_{1}\right.}^{i} \zeta_{\left.\alpha_{2} \ldots \alpha_{m}\right) \dot{\alpha}(n) i}+\bar{\nabla}_{\left(\dot{\alpha}_{1}\right.}^{i} \lambda_{\left.\alpha(m) \dot{\alpha}_{2} \ldots \dot{\alpha}_{n}\right) i}
$$

where $\zeta_{\alpha(m-1) \dot{\alpha}(n) i}$ and $\lambda_{\alpha(m) \dot{\alpha}(n-1) i}$ are complex unconstrained. 
When $n=0$, we obtain the second family of supercurrents, namely $J^{\alpha(m)}$, which satisfy (2.4). It is clear that the corresponding prepotentials, $\Upsilon_{\alpha(m)}$, are characterised by the gauge transformation law:

$$
\delta_{\zeta, \omega} \Upsilon_{\alpha(m)}=\nabla_{\left(\alpha_{1}\right.}^{i} \zeta_{\left.\alpha_{2} \ldots \alpha_{m}\right) i}+\bar{\nabla}^{i j} \omega_{\alpha(m) i j}
$$

where $\zeta_{\alpha(m-1) i}$ and $\omega_{\alpha(m) i j}$ are complex unconstrained.

Finally, for $m=n=0$, we obtain the real scalar supercurrent $J$. It is constrained by (2.6) and its dual, $\Upsilon$, may be chosen to be real and has the gauge transformation law

$$
\delta_{\omega} \Upsilon=\bar{\nabla}^{i j} \omega_{i j}+\text { c.c. }
$$

where the gauge parameter $\omega_{i j}$ is complex unconstrained. As expected, this prepotential describes the conformal supergravity multiplet.

\subsection{Superconformal models for the $\Upsilon_{\alpha(m) \dot{\alpha}(n)}$ prepotentials}

To begin with, we study the prepotentials $\Upsilon_{\alpha(m) \dot{\alpha}(n)}$ (3.1). Requiring that both these and their corresponding gauge parameters, $\zeta_{\alpha(m-1) \dot{\alpha}(n) i}$ and $\lambda_{\alpha(m) \dot{\alpha}(n-1) i}$, are superconformally primary,

$$
K^{B} \Upsilon_{\alpha(m) \dot{\alpha}(n)}=0, \quad K^{B} \zeta_{\alpha(m-1) \dot{\alpha}(n) i}=0, \quad K^{B} \lambda_{\alpha(n) \dot{\alpha}(n-1) i}=0,
$$

uniquely fixes the dimension and $\mathrm{U}(1)_{R}$ charge of $\Upsilon_{\alpha(m) \dot{\alpha}(n)}$

$$
\mathbb{D} \Upsilon_{\alpha(m) \dot{\alpha}(n)}=-\frac{1}{2}(m+n+4) \Upsilon_{\alpha(m) \dot{\alpha}(n)}, \quad Y \Upsilon_{\alpha(m) \dot{\alpha}(n)}=(m-n) \Upsilon_{\alpha(m) \dot{\alpha}(n)}
$$

This implies that for $m=n=s$, we are able to choose $\Upsilon_{\alpha(s) \dot{\alpha}(s)}$ to be real, in which case (3.1) reduces to

$$
\delta_{\zeta} \Upsilon_{\alpha(s) \dot{\alpha}(s)}=\nabla_{\left(\alpha_{1}\right.}^{i} \zeta_{\left.\alpha_{2} \ldots \alpha_{s}\right) \dot{\alpha}(s) i}-\bar{\nabla}_{\left(\dot{\alpha}_{1}\right.}^{i} \bar{\zeta}_{\left.\alpha(s) \dot{\alpha}_{2} \ldots \dot{\alpha}_{s}\right) i} .
$$

In flat superspace, (3.6) was first proposed in [1].

From the prepotential $\Upsilon_{\alpha(m) \dot{\alpha}(n)}$ and its conjugate $\bar{\Upsilon}_{\alpha(n) \dot{\alpha}(m)}$, we may construct the higher-derivative chiral descendants ${ }^{3}$

$$
\begin{aligned}
& \hat{\mathfrak{W}}_{\alpha(m+n+2)}(\Upsilon)=\bar{\nabla}^{4} \nabla_{\left(\alpha_{1}\right.}{ }_{\dot{\beta}_{1}} \ldots \nabla_{\alpha_{n}}{ }^{\dot{\beta}_{n}} \nabla_{\alpha_{n+1} \alpha_{n+2}} \Upsilon_{\left.\alpha_{n+3} \ldots \alpha_{m+n+2}\right) \dot{\beta}(n)}, \\
& \check{\mathfrak{W}}_{\alpha(m+n+2)}(\bar{\Upsilon})=\bar{\nabla}^{4} \nabla_{\left(\alpha_{1}\right.} \dot{\beta}_{1} \ldots \nabla_{\alpha_{m}}{ }^{\dot{\beta}_{m}} \nabla_{\alpha_{m+1} \alpha_{m+2}} \bar{\Upsilon}_{\left.\alpha_{m+3} \ldots \alpha_{m+n+2}\right) \dot{\beta}(m)}
\end{aligned}
$$

Here we have introduced the chiral projection operator

$$
\bar{\nabla}^{4} \equiv \frac{1}{48} \bar{\nabla}^{i j} \bar{\nabla}_{i j}
$$

and the second-order operators

$$
\nabla_{\alpha \beta}:=\nabla_{(\alpha}^{k} \nabla_{\beta) k}, \quad \bar{\nabla} \dot{\alpha} \dot{\beta}:=\bar{\nabla}_{k}^{(\dot{\alpha}} \bar{\nabla}^{\dot{\beta}) k} .
$$

\footnotetext{
${ }^{3}$ In the flat-superspace limit, these chiral field strengths reduce to those introduced in [38, 39].
} 
It should be emphasised that, for the special case $m=n=s$, the chiral descendants (3.7) coincide; $\hat{\mathfrak{W}}_{\alpha(2 s+2)}(\Upsilon)=\check{\mathfrak{W}}_{\alpha(2 s+2)}(\bar{\Upsilon}) \equiv \mathfrak{W}_{\alpha(2 s+2)}(\Upsilon)$. The chiral field strengths have the following dimensions:

$$
\begin{aligned}
& \mathbb{D} \hat{\mathfrak{W}}_{\alpha(m+n+2)}(\Upsilon)=\frac{1}{2}(n-m+2) \hat{\mathfrak{W}}_{\alpha(m+n+2)}(\Upsilon), \\
& \mathbb{D} \check{\mathfrak{W}}_{\alpha(m+n+2)}(\bar{\Upsilon})=\frac{1}{2}(m-n+2) \check{\mathfrak{W}}_{\alpha(m+n+2)}(\bar{\Upsilon}) .
\end{aligned}
$$

Further, it may be shown that they are primary,

$$
K^{B} \hat{\mathfrak{W}}_{\alpha(m+n+2)}(\Upsilon)=0, \quad K^{B} \check{\mathfrak{W}}_{\alpha(m+n+2)}(\bar{\Upsilon})=0
$$

These properties imply that the action

$$
S^{(m, n)}=\mathrm{i}^{m+n} \int \mathrm{d}^{4} x \mathrm{~d}^{4} \theta \mathcal{E} \hat{\mathfrak{W}}^{\alpha(m+n+2)}(\Upsilon) \check{\mathfrak{W}}_{\alpha(m+n+2)}(\bar{\Upsilon})+\text { c.c. }
$$

is locally superconformal. Here $\mathcal{E}$ is the chiral integration measure. Chiral and full superspace integrals are related according to the rule

$$
\int \mathrm{d}^{4} x \mathrm{~d}^{4} \theta \mathrm{d}^{4} \bar{\theta} E U=\int \mathrm{d}^{4} x \mathrm{~d}^{4} \theta \mathcal{E} \bar{\nabla}^{4} U, \quad E^{-1}=\operatorname{Ber}\left(E_{A}{ }^{M}\right),
$$

where $U$ is a real primary dimension- 0 superfield. Derivations of this result using superspace normal coordinates are given in $[19,40]$. The overall factor of $\mathrm{i}^{m+n}$ in $(3.12)$ has been chosen due to the identity

$$
\mathrm{i}^{m+n+1} \int \mathrm{d}^{4} x \mathrm{~d}^{4} \theta \mathcal{E} \hat{\mathfrak{W}}^{\alpha(m+n+2)}(\Upsilon) \check{\mathfrak{W}}_{\alpha(m+n+2)}(\bar{\Upsilon})+\text { c.c. }=0,
$$

which holds up to a total derivative for any conformally flat background (2.10).

We restrict our attention to conformally flat superspaces. In these geometries it may be shown that the chiral descendants (3.7) are gauge-invariant

$$
\delta_{\zeta, \lambda} \hat{\mathfrak{W}}_{\alpha(m+n+2)}(\Upsilon)=0, \quad \delta_{\zeta, \lambda} \check{\mathfrak{W}}_{\alpha(m+n+2)}(\bar{\Upsilon})=0
$$

As a result, the action (3.12) is gauge-invariant, thus the field strengths (3.7) are linearised higher-spin super-Weyl tensors.

\subsection{Superconformal models for the $\Upsilon_{\alpha(m)}$ prepotentials}

The gauge prepotentials $\Upsilon_{\alpha(m)}$, with $m \geq 1$ are defined modulo the transformations (3.2). Similar to the $n=0$ case, requiring that both the prepotentials and gauge parameters are superconformally primary,

$$
K^{B} \Upsilon_{\alpha(m)}=0, \quad K^{B} \zeta_{\alpha(m-1) i}=0, \quad K^{B} \omega_{\alpha(n) i j}=0,
$$

determines the dimension and $\mathrm{U}(1)_{R}$ charge of $\Upsilon_{\alpha(m)}$

$$
\mathbb{D} \Upsilon_{\alpha(m)}=-\frac{1}{2}(m+4) \Upsilon_{\alpha(m)}, \quad Y \Upsilon_{\alpha(m)}=m \Upsilon_{\alpha(m)}
$$


Associated with the prepotential $\Upsilon_{\alpha(m)}$ (and its conjugate $\bar{\Upsilon}_{\dot{\alpha}(m)}$ ) are the chiral descendants:

$$
\begin{aligned}
& \hat{\mathfrak{W}}_{\alpha(m+2)}(\Upsilon)=\bar{\nabla}^{4} \nabla_{\left(\alpha_{1} \alpha_{2}\right.} \Upsilon_{\left.\alpha_{3} \ldots \alpha_{m+2}\right)}, \\
& \check{\mathfrak{W}}_{\alpha(m+2)}(\bar{\Upsilon})=\bar{\nabla}^{4} \nabla_{\left(\alpha_{1}\right.} \dot{\beta}_{1} \ldots \nabla_{\alpha_{m}} \dot{\beta}_{m} \nabla_{\left.\alpha_{m+1} \alpha_{m+2}\right)} \bar{\Upsilon}_{\dot{\beta}(m)} .
\end{aligned}
$$

It may readily be shown that (i) their dimensions are

$$
\begin{aligned}
& \mathbb{D} \hat{\mathfrak{W}}_{\alpha(m+2)}(\Upsilon)=-\frac{1}{2}(m-2) \hat{\mathfrak{W}}_{\alpha(m+2)}(\Upsilon), \\
& \mathbb{D} \check{\mathfrak{W}}_{\alpha(m+2)}(\bar{\Upsilon})=\frac{1}{2}(m+2) \check{\mathfrak{W}}_{\alpha(m+2)}(\bar{\Upsilon}) ;
\end{aligned}
$$

and that (ii) they are primary

$$
K^{B} \hat{\mathfrak{W}}_{\alpha(m+2)}(\Upsilon)=0, \quad K^{B} \check{\mathfrak{W}}_{\alpha(m+2)}(\bar{\Upsilon})=0
$$

As a result, the following action

$$
S^{(m)}=\mathrm{i}^{m} \int \mathrm{d}^{4} x \mathrm{~d}^{4} \theta \mathcal{E} \hat{\mathfrak{W}}^{\alpha(m+2)}(\Upsilon) \check{\mathfrak{W}}_{\alpha(m+2)}(\bar{\Upsilon})+\text { c.c. }
$$

is locally superconformal. Similar to the $n \neq 0$ case, the overall coefficient of $\mathrm{i}^{m}$ has been chosen since on conformally flat backgrounds

$$
\mathrm{i}^{m+1} \int \mathrm{d}^{4} x \mathrm{~d}^{4} \theta \mathcal{E} \hat{\mathfrak{W}}^{\alpha(m+2)}(\Upsilon) \check{\mathfrak{W}}_{\alpha(m+2)}(\bar{\Upsilon})+\text { c.c. }=0,
$$

i.e. it is a total derivative.

In backgrounds with vanishing super-Weyl tensor, a routine calculation allows us to show that (3.18) are gauge-invariant field strengths

$$
\delta_{\zeta, \omega} \hat{\mathfrak{W}}_{\alpha(m+2)}(\Upsilon)=0, \quad \delta_{\zeta, \omega} \check{\mathfrak{W}}_{\alpha(m+2)}(\bar{\Upsilon})=0
$$

Thus, just as for the $n \neq 0$ case, the action (3.21) proves to be gauge-invariant and the field strengths (3.18) are linearised higher-spin super-Weyl tensors.

\subsection{Linearised $\mathcal{N}=2$ conformal supergravity}

The scalar gauge prepotential $\Upsilon=\bar{\Upsilon}$ describes linearised conformal supergravity and possesses the gauge freedom (3.3). The requirement that both $\Upsilon$ and $\omega_{i j}$ are superconformal primary,

$$
K^{B} \Upsilon=0, \quad K^{B} \omega_{i j}=0,
$$

leads to

$$
\mathbb{D} \Upsilon=-2 \Upsilon, \quad Y \Upsilon=0 .
$$

From the prepotential $\Upsilon$, we may construct the single chiral descendant

$$
\mathfrak{W}_{\alpha(2)}(\Upsilon)=\bar{\nabla}^{4} \nabla_{\alpha(2)} \Upsilon
$$


It has the following superconformal properties

$$
\mathbb{D} \mathfrak{W}_{\alpha(2)}(\Upsilon)=\mathfrak{W}_{\alpha(2)}(\Upsilon), \quad K^{B} \mathfrak{W}_{\alpha(2)}(\Upsilon)=0
$$

which imply that the following action

$$
S=\int \mathrm{d}^{4} x \mathrm{~d}^{4} \theta \mathcal{E} \mathfrak{W}^{\alpha(2)}(\Upsilon) \mathfrak{W}_{\alpha(2)}(\Upsilon)+\text { c.c. }
$$

is locally superconformal. We note that

$$
\mathrm{i} \int \mathrm{d}^{4} x \mathrm{~d}^{4} \theta \mathcal{E} \mathfrak{W}^{\alpha(2)}(\Upsilon) \mathfrak{W}_{\alpha(2)}(\Upsilon)+\text { c.c. }=0
$$

is a total derivative when $W_{\alpha \beta}=0$.

When the background geometry is conformally flat, it is easily shown that $\mathfrak{W}_{\alpha(2)}(\Upsilon)$ is invariant under the gauge transformations (3.3),

$$
\delta_{\omega} \mathfrak{W}_{\alpha(2)}(\Upsilon)=0
$$

Hence, (3.28) is gauge-invariant. Consequently, (3.26) is the linearised super-Weyl tensor.

\subsection{Reduction to $\mathcal{N}=1$ superspace}

This subsection is devoted to a discussion of the $\mathcal{N}=1$ superfield content of the real superconformal prepotentials $\Upsilon_{\alpha(s) \dot{\alpha}(s)}$ for $s \geq 0$ in conformally-flat backgrounds. ${ }^{4}$ We remind the reader that these are defined modulo the gauge transformations (3.6) for $s>0$ and (3.3) for $s=0$.

Utilising this freedom, we may choose a gauge on $\Upsilon_{\alpha(s) \dot{\alpha}(s)}$ such that the only nonvanishing $\mathcal{N}=1$ superfields in its multiplet are:

$$
\begin{aligned}
\boldsymbol{H}_{\alpha(s+1) \dot{\alpha}(s+1)} & =\frac{1}{2}\left[\nabla_{\left(\alpha_{1}\right.}, \bar{\nabla}_{\left(\dot{\alpha}_{1} \underline{2}\right.}\right] \Upsilon_{\left.\left.\alpha_{2} \ldots \alpha_{s+1}\right) \dot{\alpha}_{2} \ldots \dot{\alpha}_{s+1}\right)} \mid \\
\boldsymbol{\Psi}_{\alpha(s+1) \dot{\alpha}(s)} & =-\frac{1}{4} \nabla_{\left(\alpha_{1}\right.}\left(\bar{\nabla}_{\underline{2}}\right)^{2} \Upsilon_{\left.\alpha_{2} \ldots \alpha_{s+1}\right) \dot{\alpha}(s)} \mid \\
\boldsymbol{G}_{\alpha(s) \dot{\alpha}(s)} & =\frac{1}{32}\left\{\left(\nabla^{2}\right)^{2},\left(\bar{\nabla}_{\underline{2}}\right)^{2}\right\} \Upsilon_{\alpha(s) \dot{\alpha}(s)} \mid+\frac{1}{8(2 s+3)}\left[\nabla^{\beta}, \nabla^{\dot{\beta}}\right] \boldsymbol{H}_{\alpha(s) \beta \dot{\alpha}(s) \dot{\beta}}
\end{aligned}
$$

We emphasise that these superfields have been defined such that they are primary

$$
K^{B} \boldsymbol{H}_{\alpha(s+1) \dot{\alpha}(s+1)}=0, \quad K^{B} \boldsymbol{\Psi}_{\alpha(s+1) \dot{\alpha}(s)}=0, \quad K^{B} \boldsymbol{G}_{\alpha(s) \dot{\alpha}(s)}=0,
$$

and possess appropriate gauge freedoms; in the $s=0$ case one obtains:

$$
\begin{array}{rlrl}
\delta_{L} \boldsymbol{H}_{\alpha \dot{\alpha}} & =\bar{\nabla}_{\dot{\alpha}} L_{\alpha}-\nabla_{\alpha} \bar{L}_{\dot{\alpha}}, & & \\
\delta_{\lambda, \Lambda} \boldsymbol{\Psi}_{\alpha} & =\nabla_{\alpha} \lambda+\Lambda_{\alpha}, & \bar{\nabla}_{\dot{\alpha}} \Lambda_{\alpha}=0, \\
\delta_{\chi} \boldsymbol{G} & =\chi+\bar{\chi}, & \overline{\boldsymbol{\nabla}}_{\dot{\alpha}} \chi & =0,
\end{array}
$$

\footnotetext{
${ }^{4}$ It should be noted that for the $s=0$ case, this reduction was carried out in [41].
} 
while for $s>0$ we find:

$$
\begin{aligned}
\delta_{L} \boldsymbol{H}_{\alpha(s+1) \dot{\alpha}(s+1)} & =\bar{\nabla}_{\left(\dot{\alpha}_{1}\right.} L_{\left.\alpha(s+1) \dot{\alpha}_{2} \ldots \dot{\alpha}_{s+1}\right)}-\nabla_{\left(\alpha_{1}\right.} \bar{L}_{\left.\alpha_{2} \ldots \alpha_{s+1}\right) \dot{\alpha}(s+1)} \\
\delta_{\eta, \lambda} \boldsymbol{\Psi}_{\alpha(s+1) \dot{\alpha}(s)} & =\bar{\nabla}_{\left(\dot{\alpha}_{1}\right.} \eta_{\left.\alpha(s+1) \dot{\alpha}_{2} \ldots \dot{\alpha}_{s}\right)}+\nabla_{\left(\alpha_{1}\right.} \lambda_{\left.\alpha_{2} \ldots \alpha_{s+1}\right) \dot{\alpha}(s)} \\
\delta_{\rho} \boldsymbol{G}_{\alpha(s) \dot{\alpha}(s)} & =\bar{\nabla}_{\left(\dot{\alpha}_{1}\right.} \rho_{\left.\alpha(s) \dot{\alpha}_{2} \ldots \dot{\alpha}_{s}\right)}-\nabla_{\left(\alpha_{1} \bar{\rho}_{\left.\alpha_{2} \ldots \alpha_{s}\right) \dot{\alpha}(s)}\right.}
\end{aligned}
$$

where all gauge parameters are primary and complex unconstrained, unless otherwise stated. It should be noted that the component content of each $\mathcal{N}=1$ multiplet presented above was first reported in [3].

To conclude this section, we now reduce the action for $\Upsilon_{\alpha(s) \dot{\alpha}(s)}$

$$
S^{(s, s)}=(-1)^{s} \int \mathrm{d}^{4} x \mathrm{~d}^{4} \theta \mathcal{E} \mathfrak{W}^{\alpha(2 s+2)}(\Upsilon) \mathfrak{W}_{\alpha(2 s+2)}(\Upsilon)+\text { c.c. }
$$

from $\mathcal{N}=2$ to $\mathcal{N}=1$ conformal superspace (see also [42]). Omitting the technical details of the reduction, one arrives at the following:

$$
\begin{aligned}
S^{(s, s)}= & (-1)^{s} \int \mathrm{d}^{4} x \mathrm{~d}^{2} \theta \mathcal{E}\left\{2(2 s+2)(2 s+3) \mathfrak{W}^{\alpha(2 s+1)}(\boldsymbol{G}) \mathfrak{W}_{\alpha(2 s+1)}(\boldsymbol{G})\right. \\
& \left.+2 \mathrm{i} \hat{\mathfrak{W}}^{\alpha(2 s+2)}(\boldsymbol{\Psi}) \check{\mathfrak{W}}_{\alpha(2 s+2)}(\bar{\Psi})-\frac{1}{2} \mathfrak{W}^{\alpha(2 s+3)}(\boldsymbol{H}) \mathfrak{W}_{\alpha(2 s+3)}(\boldsymbol{H})\right\}+ \text { c.c. }
\end{aligned}
$$

where $\mathcal{E}$ denotes the $\mathcal{N}=1$ chiral integration measure and we have introduced the $\mathcal{N}=1$ higher-spin Weyl tensors:

$$
\begin{aligned}
& \hat{\mathfrak{W}}_{\alpha(m+n+1)}(\boldsymbol{\Upsilon})=-\frac{1}{4} \bar{\nabla}^{2} \nabla_{\left(\alpha_{1}\right.}{ }^{\beta_{1}} \ldots \boldsymbol{\nabla}_{\alpha_{n}}^{\beta_{n}} \boldsymbol{\nabla}_{\alpha_{n+1}} \boldsymbol{\Upsilon}_{\left.\alpha_{n+2} \ldots \alpha_{m+n+1}\right) \beta(n)}, \\
& \check{\mathfrak{W}}_{\alpha(m+n+1)}(\overline{\boldsymbol{\Upsilon}})=-\frac{1}{4} \bar{\nabla}^{2} \boldsymbol{\nabla}_{\left(\alpha_{1}\right.}{ }^{\beta_{1}} \ldots \boldsymbol{\nabla}_{\alpha_{m}}{ }^{\beta_{m}} \boldsymbol{\nabla}_{\alpha_{m+1}} \overline{\boldsymbol{\Upsilon}}_{\left.\alpha_{m+2} \ldots \alpha_{m+n+1}\right) \beta(m)},
\end{aligned}
$$

which are primary and gauge-invariant for any $\mathcal{N}=1$ SCHS gauge prepotential $\Upsilon_{\alpha(m) \dot{\alpha}(n)}$, see $[3,43]$ for the technical details. We emphasise that in the case of a real prepotential, $m=n=s$, the field strengths coincide; $\hat{\mathfrak{W}}_{\alpha(2 s+1)}(\boldsymbol{\Upsilon})=\check{\mathfrak{W}}_{\alpha(2 s+1)}(\overline{\mathbf{\Upsilon}}) \equiv \mathfrak{W}_{\alpha(2 s+1)}(\mathbf{\Upsilon})$.

\section{$4 \mathcal{N}>2$ superconformal higher-spin gauge theories}

In the above sections we have performed a systematic study of $\mathcal{N}=2$ superconformal higher-spin theories in conformally-flat backgrounds. This resulted in the construction of new families of superconformal higher-spin multiplets and their corresponding gaugeinvariant actions. Here we extend these results to the $\mathcal{N}>2$ case.

The conformal higher-spin $\mathcal{N}=2$ supercurrents and their dual gauge prepotential were introduced in the previous sections for an arbitrary conformal supergravity background. Beyond $\mathcal{N}=2$, off-shell formulations were constructed for $\mathcal{N}=3[44-46]$ and $\mathcal{N}=4[21$, $47,48]$ conformal supergravity theories. The formalism of $\mathcal{N}=4$ conformal superspace was briefly described in [48], while $\mathcal{N}=3$ conformal superspace has not yet been presented in the literature. Since currently there is no universal conformal superspace formulation for 
$\mathcal{N}$-extended conformal supergravity in four dimensions, in contrast to the three-dimensional case [49], our analysis below will be restricted to conformally flat backgrounds. ${ }^{5}$

\section{1 $\mathcal{N}>2$ conformal superspace: conformally flat case}

We consider a conformally-flat $\mathcal{N}>2$ superspace $\mathcal{M}^{4 \mid 4 \mathcal{N}}$, parametrised by local coordinates $z^{M}=\left(x^{m}, \theta_{\imath}^{\mu}, \bar{\theta}_{\dot{\mu}}^{\imath}\right)$, where $\imath=\underline{1}, \ldots, \underline{\mathcal{N}}$. Its structure group is chosen to be the $\mathcal{N}$-extended superconformal group. The latter is spanned by the Lorentz $M_{a b}$, translation $P_{A}=\left(P_{a}, Q_{\alpha}^{I}, \bar{Q}_{I}^{\dot{\alpha}}\right)$, dilatation $\mathbb{D}$, R-symmetry $Y$ and $J_{J}^{I}$, and the special conformal $K^{A}=\left(K^{a}, S_{I}^{\alpha}, \bar{S}_{\dot{\alpha}}^{I}\right)$ generators. Its geometry is encoded within the conformally covariant derivatives $\nabla_{A}=\left(\nabla_{a}, \nabla_{\alpha}^{I}, \bar{\nabla}_{I}^{\dot{\alpha}}\right)$, which take the form:

$$
\begin{aligned}
\nabla_{A} & =E_{A}-\frac{1}{2} \Omega_{A}{ }^{a b} M_{a b}-\Phi_{A}{ }^{I}{ }_{J} J_{I}^{J}-\mathrm{i} \Phi_{A} Y-B_{A} \mathbb{D}-\mathfrak{F}_{A B} K^{B} \\
& =E_{A}-\Omega_{A}{ }^{\beta \gamma} M_{\beta \gamma}-\bar{\Omega}_{A}{ }^{\dot{\beta} \dot{\gamma}} \bar{M}_{\dot{\beta} \dot{\gamma}}-\Phi_{A}{ }^{I}{ }_{J} J^{J}{ }_{I}-\mathrm{i} \Phi_{A} Y-B_{A} \mathbb{D}-\mathfrak{F}_{A B} K^{B} .
\end{aligned}
$$

The algebraic relations obeyed by the generators of the $\mathcal{N}$-extended superconformal algebra take the same functional form as in the $\mathcal{N}=2$ case (A.3) with some minor exceptions. In particular, one should recall that, for $\mathcal{N}>2$, the $\operatorname{SU}(\mathcal{N})_{R}$ generator $J^{I}{ }_{J}$ acts on isospinors as follows

$$
J_{J}^{I} \chi^{K}=-\delta_{J}^{K} \chi^{I}+\frac{1}{\mathcal{N}} \delta_{J}^{I} \chi^{K}
$$

Additionally, the $\mathrm{U}(1)_{R}$ charges of the spinor covariant derivatives and special conformal generators now take the form:

$$
\begin{aligned}
{\left[Y, \nabla_{\alpha}^{I}\right] } & =\frac{4-\mathcal{N}}{\mathcal{N}} \nabla_{\alpha}^{I}, & {\left[Y, \bar{\nabla}_{I}^{\dot{\alpha}}\right] } & =\frac{\mathcal{N}-4}{\mathcal{N}} \bar{\nabla}_{I}^{\dot{\alpha}}, \\
{\left[Y, S_{I}^{\alpha}\right] } & =\frac{\mathcal{N}-4}{\mathcal{N}} S_{I}^{\alpha}, & {\left[Y, \bar{S}_{\dot{\alpha}}^{I}\right] } & =\frac{4-\mathcal{N}}{\mathcal{N}} \bar{S}_{\dot{\alpha}}^{I} .
\end{aligned}
$$

The non-vanishing anti-commutation relations between spinor covariant derivatives and special conformal generators now take the form:

$$
\begin{aligned}
& \left\{S_{I}^{\alpha}, \nabla_{\beta}^{J}\right\}=\delta_{I}^{J} \delta_{\beta}^{\alpha}(2 \mathbb{D}-Y)-4 \delta_{I}^{J} M^{\alpha}{ }_{\beta}+4 \delta_{\beta}^{\alpha} J_{I}^{J}, \\
& \left\{\bar{S}_{\dot{\alpha}}^{I}, \bar{\nabla}_{J}^{\dot{\beta}}\right\}=\delta_{J}^{I} \delta_{\dot{\alpha}}^{\dot{\beta}}(2 \mathbb{D}+Y)+4 \delta_{J}^{I} \bar{M}_{\dot{\alpha}}{ }^{\dot{\beta}}-4 \delta_{\dot{\alpha}}^{\dot{\beta}} J^{I}{ }_{J}
\end{aligned}
$$

It should be noted that, for $\mathcal{N}=4$, the $\mathrm{U}(1)_{R}$ generator becomes a central charge commuting with all elements of the superconformal algebra, see [2] for a detailed discussion.

In general, the graded commutators $\left[\nabla_{A}, \nabla_{B}\right\}$ should be constrained in order to be expressed solely in terms of a single primary ${ }^{6}$ tensor superfield, the $\mathcal{N}$-extended super-Weyl tensor $W$ (with suppressed indices) and its covariant derivatives. In this section, we will restrict our attention to so-called conformally-flat backgrounds, which are characterised by the constraint $W=0$. As a result, the only non-vanishing sector of $\left[\nabla_{A}, \nabla_{B}\right\}$ is

$$
\left\{\nabla_{\alpha}^{I}, \bar{\nabla}_{J}^{\dot{\alpha}}\right\}=-2 \mathrm{i} \delta_{J}^{I} \nabla_{\alpha}^{\dot{\alpha}}
$$

\footnotetext{
${ }^{5}$ In the component approach, the gauging of the $\mathcal{N}$-extended superconformal group in four dimensions was studied for the first time in [50], see also [2] for a review.

${ }^{6}$ A tensor superfield $\Psi$ with suppressed indices is said to be primary if it satisfies $K^{B} \Psi=0$.
} 


\subsection{Conformal supercurrent multiplets and gauge prepotentials}

Let $m$ and $n$ be positive integers. A primary tensor superfield $\hat{J}^{\alpha(m) \dot{\alpha}(n)}$ defined on the background superspace will be called a conformal supercurrent if it obeys

$$
\begin{array}{rll}
\nabla_{\beta}^{I} \hat{J}^{\beta \alpha(m-1) \dot{\alpha}(n)}=0 & \Longrightarrow & \nabla^{I J} \hat{J}^{\alpha(m) \dot{\alpha}(n)}=0, \\
\bar{\nabla}_{\dot{\beta} I} \hat{J}^{\alpha(m) \dot{\beta} \dot{\alpha}(n-1)}=0 & \Longrightarrow & \bar{\nabla}_{I J} \hat{J}^{\alpha(m) \dot{\alpha}(n)}=0,
\end{array}
$$

where we have denoted

$$
\nabla^{I J}=\nabla^{\alpha(I} \nabla_{\alpha}^{J)}, \quad \bar{\nabla}_{I J}=\bar{\nabla}_{\dot{\alpha}(I} \bar{\nabla}_{J)}^{\dot{\alpha}} .
$$

These constraints uniquely fix the superconformal properties of $J^{\alpha(m) \dot{\alpha}(n)}$

$$
\mathbb{D} \hat{J}^{\alpha(m) \dot{\alpha}(n)}=\frac{1}{2}(m+n+4) \hat{J}^{\alpha(m) \dot{\alpha}(n)}, \quad Y \hat{J}^{\alpha(m) \dot{\alpha}(n)}=-(m-n) \hat{J}^{\alpha(m) \dot{\alpha}(n)} .
$$

When $n=0$, the constraints (4.7) should be replaced with

$$
\begin{aligned}
\nabla_{\beta}^{I} \hat{J}^{\beta \alpha(m-1)} & =0 \quad \Longrightarrow \quad \nabla^{I J} \hat{J}^{\alpha(m)}=0, \\
\bar{\nabla}_{I J} \hat{J}^{\alpha(m)} & =0 .
\end{aligned}
$$

Their consistency with the superconformal algebra implies:

$$
\mathbb{D} \hat{J}^{\alpha(m)}=\frac{1}{2}(m+4) \hat{J}^{\alpha(m)}, \quad Y \hat{J}^{\alpha(m)}=-m \hat{J}^{\alpha(m)} .
$$

Finally, for the special case $m=0$, we replace (4.10) with

$$
\nabla^{I J} \hat{J}=0, \quad \bar{\nabla}_{I J} \hat{J}=0,
$$

which imply:

$$
\mathbb{D} \hat{J}=2 \hat{J}, \quad Y \hat{J}=0 .
$$

In $\mathcal{N}$-extended Minkowski superspace $\mathbb{M}^{4 \mid 4 \mathcal{N}}$, the conformal supercurrents $\hat{J}^{\alpha(n) \dot{\alpha}(n)}$ with $n>0$ were introduced in [1]. Certain primary supermultiplets $\mathbb{M}^{4 \mid 4 \mathcal{N}}$ of the types (4.7), (4.10) and (4.12) were also defined in [51].

We now consider the Noether coupling

$$
S_{\mathrm{NC}}=\int \mathrm{d}^{4} x \mathrm{~d}^{2 \mathcal{N}} \theta \mathrm{d}^{2 \mathcal{N}} \bar{\theta} \hat{E} \hat{\Upsilon}_{\alpha(m) \dot{\alpha}(n)} \hat{J}^{\alpha(m) \dot{\alpha}(n)},
$$

where $\hat{E}$ is the full superspace measure and $\hat{\Upsilon}_{\alpha(m) \dot{\alpha}(n)}$ is the gauge prepotential dual to $\hat{J}^{\alpha(m) \dot{\alpha}(n)}$. For $m, n \geq 1,(4.14)$ is inert under the transformations

$$
\delta_{\hat{\zeta}, \hat{\lambda}} \hat{\Upsilon}_{\alpha(m) \dot{\alpha}(n)}=\nabla_{\left(\alpha_{1}\right.}^{I} \hat{\zeta}_{\left.\alpha_{2} \ldots \alpha_{m}\right) \dot{\alpha}(n) I}+\bar{\nabla}_{\left(\dot{\alpha}_{1} I\right.} \hat{\lambda}_{\left.\alpha(m) \dot{\alpha}_{2} \ldots \dot{\alpha}_{n}\right)}^{I}
$$

In the special case $n=0$, the above gauge transformation should be replaced with

$$
\delta_{\hat{\zeta}, \hat{\omega}} \hat{\Upsilon}_{\alpha(m)}=\nabla_{\left(\alpha_{1}\right.}^{I} \hat{\zeta}_{\left.\alpha_{2} \ldots \alpha_{m}\right) I}+\bar{\nabla}_{I J} \hat{\omega}_{\alpha(m)}^{I J}
$$


Finally, setting $m=n=0$, this should be replaced with

$$
\delta_{\hat{\omega}} \hat{\Upsilon}=\bar{\nabla}_{I J} \hat{\omega}^{I J}+\text { c.c. }
$$

Requiring both the prepotentials $\hat{\Upsilon}_{\alpha(m) \dot{\alpha}(n)}, m, n \geq 0$, and corresponding gauge parameters to be primary uniquely fixes the dimension and $\mathrm{U}(1)_{R}$ charge of the former:

$$
\begin{aligned}
& \mathbb{D} \hat{\Upsilon}_{\alpha(m) \dot{\alpha}(n)}=-\frac{1}{2}(m+n+4(\mathcal{N}-1)) \hat{\Upsilon}_{\alpha(m) \dot{\alpha}(n)}, \\
& Y \hat{\Upsilon}_{\alpha(m) \dot{\alpha}(n)}=(m-n) \hat{\Upsilon}_{\alpha(m) \dot{\alpha}(n)} .
\end{aligned}
$$

\subsection{Superconformal gauge-invariant models}

In this subsection we present a gauge-invariant action for each gauge prepotential derived in the previous subsection. From the superfield $\hat{\Upsilon}_{\alpha(m) \dot{\alpha}(n)}, m, n \geq 0$, we construct the higher-derivative descendants

$$
\begin{aligned}
& \hat{\mathfrak{W}}_{\alpha(m+n+\mathcal{N})}(\hat{\Upsilon})=\bar{\nabla}^{2 \mathcal{N}} \nabla_{\left(\alpha_{1}\right.} \dot{\beta}_{1} \ldots \nabla_{\alpha_{n}} \dot{\beta}_{n} \nabla_{\alpha_{n+1} \ldots \alpha_{n+\mathcal{N}}} \hat{\Upsilon}_{\alpha_{n+\mathcal{N}+1} \ldots \alpha_{m+n+\mathcal{N})}(n)}, \\
& \check{\mathfrak{W}}_{\alpha(m+n+\mathcal{N})}(\hat{\widetilde{\Upsilon}})=\bar{\nabla}^{2 \mathcal{N}} \nabla_{\left(\alpha_{1}\right.} \dot{\beta}_{1} \ldots \nabla_{\alpha_{m}}{ }^{\dot{\beta}_{m}} \nabla_{\alpha_{m+1} \ldots \alpha_{m+\mathcal{N}}} \hat{\widehat{\Upsilon}}_{\alpha_{m+\mathcal{N}+1} \ldots \alpha_{m+n+\mathcal{N})} \dot{\beta}(m)} .
\end{aligned}
$$

Here we have introduced the $\mathcal{N}$ th order operators ${ }^{7}$

$$
\nabla_{\alpha(\mathcal{N})}=\varepsilon_{I_{1} \ldots I_{\mathcal{N}}} \nabla_{\left(\alpha_{1}\right.}^{I_{1}} \ldots \nabla_{\left.\alpha_{\mathcal{N}}\right)}^{I_{\mathcal{N}}}, \quad \bar{\nabla}^{\dot{\alpha}(\mathcal{N})}=\varepsilon^{I_{1} \ldots I_{\mathcal{N}}} \bar{\nabla}_{I_{1}}^{\left(\dot{\alpha}_{1}\right.} \ldots \bar{\nabla}_{I_{\mathcal{N}}}^{\left.\dot{\alpha}_{\mathcal{N}}\right)},
$$

where $\varepsilon^{1 \ldots \mathcal{N}}=\varepsilon_{1 \ldots \mathcal{N}}=1$, as well as the chiral projection operator

$$
\bar{\nabla}^{2 \mathcal{N}}=\bar{\nabla}_{\dot{\alpha}(\mathcal{N})} \bar{\nabla}^{\dot{\alpha}(\mathcal{N})} .
$$

In the case of a Minkowski background, the field strengths (4.17) coincide with those introduced in [39].

The chiral field strengths (4.17) have the following dimensions:

$$
\begin{aligned}
& \mathbb{D} \hat{\mathfrak{W}}_{\alpha(m+n+\mathcal{N})}(\Upsilon)=\frac{1}{2}(n-m-\mathcal{N}+4) \hat{\mathfrak{W}}_{\alpha(m+n+\mathcal{N})}(\Upsilon), \\
& \mathbb{D} \check{\mathfrak{W}}_{\alpha(m+n+\mathcal{N})}(\bar{\Upsilon})=\frac{1}{2}(m-n-\mathcal{N}+4) \check{\mathfrak{W}}_{\alpha(m+n+\mathcal{N})}(\bar{\Upsilon}),
\end{aligned}
$$

and may be shown to be primary

$$
K^{B} \hat{\mathfrak{W}}_{\alpha(m+n+\mathcal{N})}(\Upsilon)=0, \quad K^{B} \check{\mathfrak{W}}_{\alpha(m+n+\mathcal{N})}(\bar{\Upsilon})=0,
$$

and are invariant under the gauge transformations (4.15).

These properties imply that the action

$$
S^{(m, n)}=\mathrm{i}^{m+n} \int \mathrm{d}^{4} x \mathrm{~d}^{2 \mathcal{N}} \theta \hat{\mathcal{E}} \hat{\mathfrak{W}}^{\alpha(m+n+\mathcal{N})}(\Upsilon) \check{\mathfrak{W}}_{\alpha(m+n+\mathcal{N})}(\bar{\Upsilon})+\text { c.c. }
$$

is locally superconformal and gauge-invariant. Here $\hat{\mathcal{E}}$ is the chiral integration measure. The overall factor of $\mathrm{i}^{m+n}$ in (3.12) has been chosen due to the identity

$$
\mathrm{i}^{m+n+1} \int \mathrm{d}^{4} x \mathrm{~d}^{4} \theta \hat{\mathcal{E}} \hat{\mathfrak{W}}^{\alpha(m+n+\mathcal{N})}(\Upsilon) \check{\mathfrak{W}}_{\alpha(m+n+\mathcal{N})}(\bar{\Upsilon})+\text { c.c. }=0
$$

which holds up to a total derivative.

\footnotetext{
${ }^{7}$ For simplicity, we do not include any numerical factors in (4.18) and (4.19).
} 


\section{Conclusion}

In this work we have, for the first time, introduced the $\mathcal{N}=2$ superconformal higher-spin gauge prepotentials $\Upsilon_{\alpha(m) \dot{\alpha}(n)}$ in conformal superspace, with $m \geq n \geq 0$, and derived their gauge-invariant actions in arbitrary conformally flat backgrounds. ${ }^{8}$ These results have also been generalised to $\mathcal{N}>2$. In both $\mathcal{N}=2$ and $\mathcal{N}>2$ cases, the superconformal higherspin gauge prepotentials originate as the duals of the superconformal higher-spin multiplets of conserved currents, which have been described in our paper.

Our work provides the stepping stones to address several interesting problems. First of all, it would be interesting to extend some of our $\mathcal{N}=2$ superconformal gauge models to non-conformally flat supergravity backgrounds. ${ }^{9}$ In particular, there must exist a consistent deformation of the linearised action for $\mathcal{N}=2$ conformal supergravity (3.28) to arbitrary Bach-flat backgrounds described by eq. (A.12).

Our $\mathcal{N}=2$ superconformal higher-spin models have already been demonstrated to be $\mathrm{U}(1)$ duality invariant [42]. It would be interesting to analyse whether their $\mathcal{N}>2$ cousins constructed in section 4 share this remarkable property.

It is of interest to study the problem of using Vasiliev's unfolded dynamics formalism [56] in order to reconstruct our superspace formulations directly from the component ones along the lines of the approach of [57]. The latter can be applied to the construction of conformal higher-spin currents of [58] uplifted to arbitrary conformally flat background. These issues will be addressed elsewhere.

In the remainder of this section we will concentrate, in some more detail, on several implications and natural extensions of our results.

\section{1 $\mathcal{N}=2$ superconformal higher-spin gauge theories in $\mathbf{A d S}_{4}$}

Fundamental to our analysis is the formalism of conformal superspace, which trivialises calculations in conformally flat backgrounds. For the purpose of applications, however, it is often useful to work with Lorentz covariant derivatives as opposed to their conformally covariant counterparts. The process of translating results from conformal to $\mathrm{U}(2)$ superspace is known as degauging [19] and while the general procedure is well-known, it is often highly non-trivial to perform on generic curved backgrounds. Such computations are greatly simplified when we restrict the geometry by turning off several torsion superfields.

To this end, we now degauge and reiterate our main results in the $\mathcal{N}=2$ AdS superspace. ${ }^{10}$ Recall that its geometry is encoded within the covariant derivatives $\mathcal{D}_{A}=$ $\left(\mathcal{D}_{a}, \mathcal{D}_{\alpha}^{i}, \overline{\mathcal{D}}_{i}^{\dot{\alpha}}\right)$, which obey the algebra (see, e.g. $\left.[23,60]\right)$ :

$$
\left\{\mathcal{D}_{\alpha}^{i}, \mathcal{D}_{\beta}^{j}\right\}=4 S^{i j} M_{\alpha \beta}+2 \varepsilon_{\alpha \beta} \varepsilon^{i j} S^{k l} J_{k l}, \quad\left\{\mathcal{D}_{\alpha}^{i}, \overline{\mathcal{D}}_{j}^{\dot{\beta}}\right\}=-2 \mathrm{i} \delta_{j}^{i} \mathcal{D}_{\alpha}^{\dot{\beta}},
$$

\footnotetext{
${ }^{8}$ It should be emphasised once more that the actions (3.12), (3.21) and (3.28) are locally superconformal in any curved background. But they are gauge invariant only if the background super-Weyl tensor vanishes.

${ }^{9}$ The construction of gauge-invariant models for conformal higher-spin (super)fields in curved (super)gravity backgrounds has been an active area of research [3, 4, 6, 7, 43, 52-55].

${ }^{10}$ The conformal flatness of $\mathcal{N}$-extended AdS superspace in four dimensions was established in [59].
} 
where $S^{i j}$ is a covariantly constant iso-triplet, $S^{j i}=S^{i j}$, satisfying the integrability condition $\left[S, S^{\dagger}\right]=0$, with $S=\left(S^{i}{ }_{j}\right) \cdot{ }^{11}$

We first derived the $\mathcal{N}=2$ conformal supercurrents, which are described by the tensor superfields $J^{\alpha(m) \dot{\alpha}(n)}$ with $m, n \geq 0$. For $m, n \geq 1$, they obey:

$$
\begin{aligned}
& \mathcal{D}_{\beta}^{i} J^{\beta \alpha(m-1) \dot{\alpha}(n)}=0 \quad \Longrightarrow \quad\left(\mathcal{D}^{i j}+2(m+2) S^{i j}\right) J^{\alpha(m) \dot{\alpha}(n)}=0, \\
& \overline{\mathcal{D}}_{\dot{\beta}}^{i} J^{\alpha(m) \dot{\beta} \dot{\alpha}(n-1)}=0 \quad \Longrightarrow \quad\left(\overline{\mathcal{D}}^{i j}+2(n+2) \bar{S}^{i j}\right) J^{\alpha(m) \dot{\alpha}(n)}=0,
\end{aligned}
$$

where we have defined $\mathcal{D}^{i j}=\mathcal{D}^{\alpha(i} \mathcal{D}_{\alpha}^{j)}$ and $\overline{\mathcal{D}}^{i j}=\overline{\mathcal{D}}_{\dot{\alpha}}^{(i} \overline{\mathcal{D}}^{\dot{\alpha} j)}$. When $n=0$ (5.2) should be replaced with

$$
\begin{aligned}
\mathcal{D}_{\beta}^{i} J^{\beta \alpha(m-1)} & =0 \quad \Longrightarrow \quad\left(\mathcal{D}^{i j}+2(m+2) S^{i j}\right) J^{\alpha(m)}=0, \\
\left(\overline{\mathcal{D}}^{i j}+4 \bar{S}^{i j}\right) J^{\alpha(m)} & =0
\end{aligned}
$$

Finally, for $m=n=0$ we exchange (5.3) with

$$
\left(\mathcal{D}^{i j}+4 S^{i j}\right) J=0, \quad\left(\overline{\mathcal{D}}^{i j}+4 \bar{S}^{i j}\right) J=0 .
$$

The supercurrents described above are dual, via the Noether coupling (1.1), to gauge prepotentials $\Upsilon_{\alpha(m) \alpha(n)}$, with $m, n \geq 0$. For $m, n \geq 1$, they are defined modulo the gauge transformations

$$
\delta_{\zeta, \lambda} \Upsilon_{\alpha(m) \dot{\alpha}(n)}=\mathcal{D}_{\left(\alpha_{1}\right.}^{i} \zeta_{\left.\alpha_{2} \ldots \alpha_{m}\right) \dot{\alpha}(n) i}+\overline{\mathcal{D}}_{\left(\dot{\alpha}_{1}\right.}^{i} \lambda_{\left.\alpha(m) \dot{\alpha}_{2} \ldots \dot{\alpha}_{n}\right) i}
$$

Here the parameters $\zeta_{\alpha(m-1) \dot{\alpha}(n) i}$ and $\lambda_{\alpha(m) \dot{\alpha}(n-1) i}$ are complex unconstrained. When $n=0$ the gauge transformation law becomes

$$
\delta_{\zeta, \omega} \Upsilon_{\alpha(m)}=\mathcal{D}_{\left(\alpha_{1}\right.}^{i} \zeta_{\left.\alpha_{2} \ldots \alpha_{m}\right) i}+\left(\overline{\mathcal{D}}^{i j}+4 \bar{S}^{i j}\right) \omega_{\alpha(m) i j}
$$

Here $\zeta_{\alpha(m-1) i}$ and $\omega_{\alpha(m) i j}$ are complex unconstrained. Finally, for $m=0$ the gauge transformation law is

$$
\delta_{\omega} \Upsilon=\left(\overline{\mathcal{D}}^{i j}+4 \bar{S}^{i j}\right) \omega_{i j}+\text { c.c. },
$$

where $\omega_{i j}$ is complex unconstrained. This prepotential describes the conformal supergravity multiplet.

Associated with $\Upsilon_{\alpha(m) \dot{\alpha}(n)}$ (and its conjugate $\bar{\Upsilon}_{\alpha(n) \dot{\alpha}(m)}$ ) are the field strengths

$$
\begin{aligned}
\hat{\mathfrak{W}}_{\alpha(m+n+2)}(\Upsilon)= & \frac{1}{48}\left(\overline{\mathcal{D}}^{i j}+4 \bar{S}^{i j}\right) \overline{\mathcal{D}}_{i j} \mathcal{D}_{\left(\alpha_{1}\right.} \dot{\beta}_{1} \ldots \mathcal{D}_{\alpha_{n}}{ }^{\dot{\beta}_{n}} \\
& \times \mathcal{D}_{\alpha_{n+1} \alpha_{n+2}} \Upsilon_{\left.\alpha_{n+3} \ldots \alpha_{m+n+2}\right) \dot{\beta}(n)}, \\
\check{\mathfrak{W}}_{\alpha(m+n+2)}(\bar{\Upsilon})= & \frac{1}{48}\left(\overline{\mathcal{D}}^{i j}+4 \bar{S}^{i j}\right) \overline{\mathcal{D}}_{i j} \mathcal{D}_{\left(\alpha_{1}\right.} \dot{\beta}_{1} \ldots \mathcal{D}_{\alpha_{m}} \dot{\beta}_{m} \\
& \times \mathcal{D}_{\alpha_{m+1} \alpha_{m+2}} \bar{\Upsilon}_{\left.\alpha_{m+3} \ldots \alpha_{m+n+2}\right) \dot{\beta}(m)},
\end{aligned}
$$

which are the linearised higher-spin super-Weyl tensors. As a final step, they may be employed to construct the superconformal gauge-invariant actions

$$
S^{(m, n)}=\mathrm{i}^{m+n} \int \mathrm{d}^{4} x \mathrm{~d}^{4} \theta \mathcal{E} \hat{\mathfrak{W}}^{\alpha(m+n+2)}(\Upsilon) \check{\mathfrak{W}}_{\alpha(m+n+2)}(\bar{\Upsilon})+\text { c.c. }
$$

\footnotetext{
${ }^{11}$ The integrability condition implies that $S^{i j}$ can be chosen to be real, $\overline{S^{i j}}=S_{i j}=\varepsilon_{i k} \varepsilon_{j l} S^{k l}$. However we will not impose the reality condition.
} 


\section{$5.2 \mathcal{N}=2$ superconformal higher-spin models as induced actions}

An interesting problem is to derive the $\mathcal{N}=2$ superconformal higher-spin models proposed in section 3 as induced actions, in the spirit of the non-supersymmetric studies carried out in [54, 61-64]. One possible approach is to couple an off-shell superconformal hypermultiplet to background gauge prepotentials $\Upsilon_{\alpha(s) \dot{\alpha}(s)}=\bar{\Upsilon}_{\alpha(s) \dot{\alpha}(s)}$ using an action of the form

$$
S[q, \bar{q} ; \Upsilon]=S_{\text {hyper }}[q, \bar{q}]+\sum_{s=0}^{\infty} \int \mathrm{d}^{4} x \mathrm{~d}^{4} \theta \mathrm{d}^{4} \bar{\theta} E \Upsilon_{\alpha(s) \dot{\alpha}(s)} J^{\alpha(s) \dot{\alpha}(s)}
$$

where $S_{\text {hyper }}$ denotes an off-shell action for the superconformal massless hypermultiplet $q$, and $J^{\alpha(s) \dot{\alpha}(s)}$ conserved higher-spin supercurrents in the model $S_{\text {hyper. }}$ Then it is natural to consider the generating functional for correlation functions of these conserved higher spin supercurrents defined by

$$
\mathrm{e}^{\mathrm{i} \Gamma[\Upsilon]}=\int \mathcal{D} q \mathcal{D} \bar{q} \mathrm{e}^{\mathrm{i} S[q, \bar{q} ; \Upsilon]}
$$

Integrating out the hypermultiplet superfields $q$ and $\bar{q}$ and computing the logarithmically divergent part of the effective action, one is expected to end up with the $\mathcal{N}=2$ superconformal higher-spin models described in section $3 .{ }^{12}$ There exist two powerful superspace approaches which offer off-shell formulations for the charged hypermultiplet: the harmonic superspace $[65,66]$ and the projective superspace [67-69]. ${ }^{13}$ Both formulations can be used to do the path integral (5.11).

It remains to comment on the higher-spin supercurrents in (5.10). For this we consider an on-shell hypermultiplet, which is described by the primary isospinor $q^{i}$ (and its conjugate $\left.\bar{q}_{i}\right)$ subject to the constraints

$$
\nabla_{\alpha}^{(i} q^{j)}=0, \quad \bar{\nabla}_{\dot{\alpha}}^{(i} q^{j)}=0
$$

These are consistent with the superconformal algebra provided

$$
\mathbb{D} q^{i}=q^{i}, \quad Y q^{i}=0
$$

The hypermultiplet supercurrent is

$$
J=-\frac{1}{2} q^{i} \bar{q}_{i}
$$

see [29] for more details. The hypermultiplet higher-spin supercurrents $J^{\alpha(s) \dot{\alpha}(s)}$, with

\footnotetext{
${ }^{12}$ This proposal is analogous to the one put forward in the $\mathcal{N}=1$ superconformal case [3].

${ }^{13}$ See [70] for a brief review of these approaches and their relationship.
} 
$s>0$, are uniquely obtained as the following composites of $q^{i}$ and $\bar{q}_{i}$ :

$$
\begin{aligned}
J^{\alpha(s) \dot{\alpha}(s)}= & \mathrm{i}^{s} \sum_{k=0}^{s}(-1)^{k}\left(\begin{array}{l}
s \\
k
\end{array}\right)^{2} \nabla^{\left(\alpha _ { 1 } \left(\dot{\alpha}_{1}\right.\right.} \ldots \nabla^{\alpha_{k} \dot{\alpha}_{k}} q^{i} \nabla^{\alpha_{k+1} \dot{\alpha}_{k+1}} \ldots \nabla^{\left.\left.\alpha_{s}\right) \dot{\alpha}_{s}\right)} \bar{q}_{i} \\
& -\frac{\mathrm{i}^{s+1}}{8} \sum_{k=0}^{s-1}(-1)^{k}\left(\begin{array}{c}
s \\
k
\end{array}\right)\left(\begin{array}{c}
s \\
k+1
\end{array}\right) \\
& \times\left\{\nabla^{\left(\alpha _ { 1 } \left(\dot{\alpha}_{1}\right.\right.} \ldots \nabla^{\alpha_{k} \dot{\alpha}_{k}} \nabla^{\alpha_{k+1} i} q_{i} \nabla^{\alpha_{k+2} \dot{\alpha}_{k+1}} \ldots \nabla^{\left.\alpha_{s}\right) \dot{\alpha}_{s-1}} \bar{\nabla}^{\left.\dot{\alpha}_{s}\right) j} \bar{q}_{j}\right. \\
& \left.-\nabla^{\left(\alpha _ { 1 } \left(\dot{\alpha}_{1}\right.\right.} \ldots \nabla^{\alpha_{k} \dot{\alpha}_{k}} \bar{\nabla}^{\dot{\alpha}_{k+1} i} q_{i} \nabla^{\alpha_{k+1} \dot{\alpha}_{k+2}} \ldots \nabla^{\left.\alpha_{s-1} \dot{\alpha}_{s}\right)} \nabla^{\left.\alpha_{s}\right) j} \bar{q}_{j}\right\} .
\end{aligned}
$$

It is instructive to verify that (5.15) does indeed satisfy the conservation equations (2.1) in any conformally flat background.

To connect our results to the existing literature on superconformal higher-spin multiplets, it is instructive to comment on the reduction of (5.10) to $\mathcal{N}=1$ superspace. First, we recall that at the $\mathcal{N}=1$ level the on-shell hypermultiplet is described by two free, massless chiral scalars:

$$
\begin{array}{ccc}
\Phi_{(+)}=\bar{q}_{\underline{1}} \mid, & \bar{\nabla}_{\dot{\alpha}} \Phi_{(+)}=0, & \nabla^{2} \Phi_{(+)}=0, \\
\Phi_{(-)}=q^{\underline{2}} \mid, & \bar{\nabla}_{\dot{\alpha}} \Phi_{(-)}=0, & \nabla^{2} \Phi_{(-)}=0 .
\end{array}
$$

This result, combined with definitions (2.16), allows one to prove that the $\mathcal{N}=1$ supercurrent multiplets encoded within (5.15) coincide with those of [3]. This, in addition to the results of section 3.4 , allows us to reduce (5.10) to $\mathcal{N}=1$ superspace

$$
\begin{aligned}
S\left[\Phi_{( \pm)} ; \boldsymbol{H}, \Psi, \boldsymbol{G}\right]= & S_{\text {hyper }}\left[\Phi_{( \pm)}\right]+\sum_{s=0}^{\infty} \int \mathrm{d}^{4} x \mathrm{~d}^{2} \theta \mathrm{d}^{2} \bar{\theta} E\left\{\frac{1}{4} \boldsymbol{H}_{\alpha(s+1) \dot{\alpha}(s+1)} j^{\alpha(s+1) \dot{\alpha}(s+1)}\right. \\
& \left.+\frac{1}{2} \boldsymbol{\Psi}_{\alpha(s+1) \dot{\alpha}(s)} j^{\alpha(s+1) \dot{\alpha}(s)}-\frac{1}{2} \overline{\boldsymbol{\Psi}}_{\alpha(s) \dot{\alpha}(s+1)} \bar{j}^{\alpha(s) \dot{\alpha}(s+1)}+\boldsymbol{G}_{\alpha(s) \dot{\alpha}(s)} j^{\alpha(s) \dot{\alpha}(s)}\right\} .
\end{aligned}
$$

Upon integrating out the chiral superfields $\Phi_{( \pm)}$and extracting the logarithmically divergent part of the effective action, we expect to arrive at the models described by (3.36).

\section{$5.3 \mathcal{N}=2$ superconformal vector and gravitino multiplets}

All chiral field strengths $\hat{\mathfrak{W}}_{\alpha(k)}$ and $\check{\mathfrak{W}}_{\alpha(k)}$ constructed in section 3 carry at least two spinor indices, $k \geq 2$. The case $k=0$ correspond to the massless vector multiplet [71] which can be described in terms of the curved superspace analogue of Mezincescu's prepotential [72] (see also [1]), $V_{i j}=V_{j i}$. The latter is a primary unconstrained real $\mathrm{SU}(2)$ triplet of dimension -2 . The expression for $\mathfrak{W}$ in terms of $V_{i j}$ is (see [73] for the derivation)

$$
\mathfrak{W}(V)=\frac{1}{4} \bar{\nabla}^{4} \nabla^{i j} V_{i j},
$$

and it defines a primary reduced chiral superfield of dimension +1 ,

$$
\nabla^{i j} \mathfrak{W}=\bar{\nabla}^{i j} \overline{\mathfrak{W}}
$$


The field strength $\mathfrak{W}$ is invariant under gauge transformations of the form

$$
\delta V^{i j}=\nabla_{k}^{\alpha} \Lambda_{\alpha}{ }^{k i j}+\bar{\nabla}_{\dot{\alpha} k} \bar{\Lambda}^{\dot{\alpha} k i j}, \quad \Lambda_{\alpha}{ }^{k i j}=\Lambda_{\alpha}{ }^{(k i j)},
$$

with $\Lambda_{\alpha}{ }^{k i j}$ being primary and unconstrained modulo the algebraic condition given.

It would be interesting to understand what is a gauge prepotential corresponding to the chiral field strengths $\hat{\mathfrak{W}}_{\alpha}$ and $\check{\mathfrak{W}}_{\alpha}$. These field strengths should correspond to a superconformal gravitino multiplet.

It should be pointed out that the multiplet of currents associated with Mezincescu's prepotential is the so-called linear multiplet $[74-76], J^{i j}$, which is a primary real iso-triplet of dimensions +2 constrained by

$$
\nabla_{\alpha}^{(i} J^{j k)}=\bar{\nabla}_{\dot{\alpha}}^{(i} J^{j k)}=0
$$

in the hypermultiplet case $\left.J^{i j}=\mathrm{i} \bar{q}^{(i} q^{j}\right)$. Strictly speaking, the action (5.10) should include an additional term, $\int \mathrm{d}^{4} x \mathrm{~d}^{4} \theta \mathrm{d}^{4} \bar{\theta} E V_{i j} J^{i j}$.

\subsection{Massless higher-spin $\mathcal{N}=2$ supermultiplets in $\mathrm{AdS}_{4}$}

It is known that massless higher-spin gauge models can be realised in terms of a conformal prepotentials coupled to lower-spin compensators. In particular, this is true of the non-supersymmetric (Fang-)Fronsdal actions in $\mathrm{AdS}_{4}[77,78]$, as well as their $\mathcal{N}=1$ supersymmetric counterparts $[79,80]$. In the present work we have identified the $\mathcal{N}=2$ superconformal higher-spin gauge prepotentials, which opens up the opportunity to construct off-shell massless higher-spin $\mathcal{N}=2$ supermultiplets in a manifestly $\operatorname{OSp}(2 \mid 4)$ invariant setting. ${ }^{14}$ So far, compensating superfields and gauge-invariant actions have been found only for linearised $\mathcal{N}=2$ supergravity [83]. Nevertheless, our construction naturally lends itself to the description of on-shell massless higher-spin supermultiplets that should originate in the massless higher-spin supersymmetric models as the only gauge-invariant field strengths which survive on the mass shell. In the $\mathcal{N}=2$ AdS superspace, they are described by primary tensor superfields $\mathbb{W}_{\alpha(k)}$, with $k \geq 1$, satisfying the constraints

$$
\overline{\mathcal{D}}_{\dot{\alpha}}^{i} \mathbb{W}_{\alpha(k)}=0, \quad \mathcal{D}^{\beta i} \mathbb{W}_{\alpha(k-1) \beta}=0
$$

These equations furnish a representation of the $\mathcal{N}=2$ superconformal group, since they are the degauged form of the following equations in conformal superspace

$$
\bar{\nabla}_{\dot{\alpha}}^{i} \mathbb{W}_{\alpha(k)}=0, \quad \nabla^{\beta i} \mathbb{W}_{\alpha(k-1) \beta}=0
$$

These are conformally invariant provided the primary chiral superfield $\mathbb{W}_{\alpha(k)}$ is characterised by the dimension

$$
\mathbb{D} \mathbb{W}_{\alpha(k)}=\frac{1}{2}(k+2) \mathbb{W}_{\alpha(k)}
$$

\footnotetext{
${ }^{14}$ In terms of $\mathcal{N}=1$ superfields, the off-shell massless higher-spin $\mathcal{N}=2$ supersymmetric gauge models in $\mathrm{AdS}_{4}$ were constructed in [81, 82].
} 
It is worth considering in more detail the case of an even $k$. Given a non-negative integer $s, \mathbb{W}_{\alpha(2 s+2)}$ is constructed in terms of a real prepotential $\mathbb{H}_{\alpha(s) \dot{\alpha}(s)}$ according to

$$
\begin{aligned}
\mathbb{W}_{\alpha(2 s+2)}(\mathbb{H})= & \frac{1}{48}\left(\overline{\mathcal{D}}^{i j}+4 \bar{S}^{i j}\right) \overline{\mathcal{D}}_{i j} \mathcal{D}_{\left(\alpha_{1}\right.} \dot{\beta}_{1} \ldots \mathcal{D}_{\alpha_{s}} \dot{\beta}_{s} \\
& \times \mathcal{D}_{\alpha_{s+1} \alpha_{s+2}} \mathbb{H}_{\left.\alpha_{s+3} \ldots \alpha_{2 s+2}\right) \dot{\beta}_{1} \ldots \dot{\beta}_{s}},
\end{aligned}
$$

which should be compared with (5.8). This field strength is invariant under the gauge transformations

$$
\delta_{\zeta} \mathbb{H}_{\alpha(s) \dot{\alpha}(s)}=\mathcal{D}_{\left(\alpha_{1}\right.}^{i} \zeta_{\left.\alpha_{2} \ldots \alpha_{s}\right) \dot{\alpha}(s) i}-\overline{\mathcal{D}}_{\left(\dot{\alpha}_{1}\right.}^{i} \bar{\zeta}_{\left.\alpha(s) \dot{\alpha}_{2} \ldots \dot{\alpha}_{s}\right) i}
$$

This gauge symmetry is similar to (3.6), which allows us to refer to $\mathbb{H}_{\alpha(s) \dot{\alpha}(s)}$ as the conformal prepotential for a massless theory. ${ }^{15}$ However, this terminology is not quite precise, since $\mathbb{H}_{\alpha(s) \dot{\alpha}(s)}$ is not a primary superfield. This follows from the fact that the dimension of $\mathbb{W}_{\alpha(2 s+2)}$ is $s+2$, eq. (5.24), while the dimension of $\mathfrak{W}_{\alpha(2 s+2)}(\Upsilon)$ is equal to +1 , eq. (3.10), for real $\Upsilon_{\alpha(s) \dot{\alpha}(s)}$.

It is instructive to comment on the $\mathcal{N}=1$ superfield content of these supermultiplets. In particular, the independent components are

$$
\begin{aligned}
\mathcal{F}_{\alpha(k)} & =\mathbb{W}_{\alpha(k)} \mid, \\
\mathcal{G}_{\alpha(k+1)} & \left.=\mathcal{D}_{\left(\alpha_{1}\right.}^{2} \mathbb{W}_{\left.\alpha_{2} \ldots \alpha_{k+1}\right)}\right),
\end{aligned}
$$

which prove to be on-shell massless $\mathcal{N}=1$ conformal superfields

$$
\begin{aligned}
\overline{\mathcal{D}}_{\dot{\alpha}} \mathcal{F}_{\alpha(k)} & =0, & \mathcal{D}^{\beta} \mathcal{F}_{\alpha(k-1) \beta} & =0, \\
\overline{\mathcal{D}}_{\dot{\alpha}} \mathcal{G}_{\alpha(k+1)} & =0, & \mathcal{D}^{\beta} \mathcal{G}_{\alpha(k) \beta} & =0,
\end{aligned}
$$

where $\mathcal{D}_{A}$ are the covariant derivatives of the $\mathcal{N}=1$ AdS superspace, see e.g. [84]. These on-shell $\mathcal{N}=1$ supermultiplets naturally occur in the $\mathcal{N}=1$ massless higher-spin gauge theories $[79,85]$.

\section{Acknowledgments}

We thank the referees for their useful suggestions. The work of SK is supported in part by the Australian Research Council, project No. DP200101944. The work of ER is supported by the Hackett Postgraduate Scholarship UWA, under the Australian Government Research Training Program.

\section{A $\mathcal{N}=2$ conformal superspace in four dimensions}

This appendix reviews $\mathcal{N}=2$ conformal superspace, a formulation for off-shell $\mathcal{N}=2$ conformal supergravity developed by Butter [19] and then reformulated in [86]. Our spinor conventions are those of [84], which are similar to [87].

\footnotetext{
${ }^{15}$ The massless theory also involves some compensating multiplets. They do not appear in the field strength (5.25).
} 
We consider a curved $\mathcal{N}=2$ superspace $\mathcal{M}^{4 \mid 8}$ parametrised by local coordinates $z^{M}=$ $\left(x^{m}, \theta_{\imath}^{\mu}, \bar{\theta}_{\dot{\mu}}^{\imath}\right)$, where $m=0,1,2,3, \mu=1,2$ and $\imath=\underline{1}, \underline{2}$. The structure group is chosen to be $\mathrm{SU}(2,2 \mid 2)$. The corresponding superalgebra is spanned by the Lorentz $M_{a b}$, translation $P_{A}=\left(P_{a}, Q_{\alpha}^{i}, \bar{Q}_{i}^{\dot{\alpha}}\right)$, dilatation $\mathbb{D}$, R-symmetry $Y$ and $J^{i j}$, and the special conformal $K^{A}=$ $\left(K^{a}, S_{i}^{\alpha}, \bar{S}_{\dot{\alpha}}^{i}\right)$ generators. The covariant derivatives $\nabla_{A}=\left(\nabla_{a}, \nabla_{\alpha}^{i}, \bar{\nabla}_{i}^{\dot{\alpha}}\right)$ then have the form

$$
\begin{aligned}
\nabla_{A} & =E_{A}-\frac{1}{2} \Omega_{A}^{a b} M_{a b}-\Phi_{A}^{i j} J_{i j}-\mathrm{i} \Phi_{A} Y-B_{A} \mathbb{D}-\mathfrak{F}_{A B} K^{B} \\
& =E_{A}-\Omega_{A}{ }^{\beta \gamma} M_{\beta \gamma}-\bar{\Omega}_{A} \dot{\beta} \dot{\gamma} \bar{M}_{\dot{\beta} \dot{\gamma}}-\Phi_{A}{ }^{i j} J_{i j}-\mathrm{i} \Phi_{A} Y-B_{A} \mathbb{D}-\mathfrak{F}_{A B} K^{B} .
\end{aligned}
$$

Here $E_{A}=E_{A}^{M} \partial_{M}$ is the supervielbein, $\Omega_{A}^{a b}$ the Lorentz connection, and $\Phi_{A}{ }^{i j}$ and $\Phi_{A}$ are the $\mathrm{SU}(2)_{R}$ and $\mathrm{U}(1)_{R}$ connections, respectively. In addition, we have a dilatation connection $B_{A}$ and a special superconformal connection $\mathfrak{F}_{A B}$.

The Lorentz $\left(M_{a b}\right)$ and $\mathrm{SU}(2)_{R}\left(J^{i j}\right)$ generators are defined to act on Weyl spinors, vectors and isospinors in the following way:

$$
\begin{aligned}
M_{\alpha \beta} \psi_{\gamma} & =\varepsilon_{\gamma(\alpha} \psi_{\beta}, & \bar{M}_{\dot{\alpha} \dot{\beta}} \bar{\psi}_{\dot{\gamma}} & =\varepsilon_{\dot{\gamma}(\dot{\alpha}} \psi_{\dot{\beta})}, \\
M_{a b} V_{c} & =2 \eta_{c[a} V_{b]}, & J^{i j} \chi^{k} & =\varepsilon^{k(i} \chi^{j)} .
\end{aligned}
$$

The $\mathrm{U}(1)_{R}$ and dilatation generators obey:

$$
\begin{array}{lll}
{\left[Y, \nabla_{\alpha}^{i}\right]=\nabla_{\alpha}^{i},} & {\left[Y, \bar{\nabla}_{i}^{\dot{\alpha}}\right]=-\bar{\nabla}_{i}^{\dot{\alpha}},} & \\
{\left[\mathbb{D}, \nabla_{a}\right]=\nabla_{a},} & {\left[\mathbb{D}, \nabla_{\alpha}^{i}\right]=\frac{1}{2} \nabla_{\alpha}^{i},} & {\left[\mathbb{D}, \bar{\nabla}_{i}^{\dot{\alpha}}\right]=\frac{1}{2} \bar{\nabla}_{i}^{\dot{\alpha}} .}
\end{array}
$$

At the same time, the special superconformal generators $K^{A}=\left(K^{a}, S_{i}^{\alpha}, \bar{S}_{\dot{\alpha}}^{i}\right)$ carry opposite $\mathrm{U}(1)_{R}$ and dilatation weight to $\nabla_{A}$ :

$$
\begin{array}{ll}
{\left[Y, S_{i}^{\alpha}\right]=-S_{i}^{\alpha},} & {\left[Y, \bar{S}_{\dot{\alpha}}^{i}\right]=\bar{S}_{\dot{\alpha}}^{i},} \\
{\left[\mathbb{D}, K_{a}\right]=-K_{a}, \quad\left[\mathbb{D}, S_{i}^{\alpha}\right]=-\frac{1}{2} S_{i}^{\alpha}, \quad\left[\mathbb{D}, \bar{S}_{\dot{\alpha}}^{i}\right]=-\frac{1}{2} \bar{S}_{\dot{\alpha}}^{i}}
\end{array}
$$

Among themselves, the generators $K^{A}$ obey the algebra

$$
\left\{S_{i}^{\alpha}, \bar{S}_{\dot{\alpha}}^{j}\right\}=2 \mathrm{i} \delta_{i}^{j} K_{\dot{\alpha}}^{\alpha},
$$

with all the other (anti-)commutators vanishing. Finally, the algebra of $K^{A}$ with $\nabla_{B}$ is given by

$$
\begin{aligned}
{\left[K_{\alpha \dot{\alpha}}, \nabla_{\beta \dot{\beta}}\right] } & =-4 \varepsilon_{\alpha \beta} \varepsilon_{\dot{\alpha} \dot{\beta}} \mathbb{D}+4 \varepsilon_{\dot{\alpha} \dot{\beta}} M_{\alpha \beta}+4 \varepsilon_{\alpha \beta} \bar{M}_{\dot{\alpha} \dot{\beta}}, \\
\left\{S_{i}^{\alpha}, \nabla_{\beta}^{j}\right\} & =\delta_{i}^{j} \delta_{\beta}^{\alpha}(2 \mathbb{D}-Y)-4 \delta_{i}^{j} M^{\alpha}{ }_{\beta}+4 \delta_{\beta}^{\alpha} J_{i}{ }^{j}, \\
\left\{\bar{S}_{\dot{\alpha}}^{i}, \bar{\nabla}_{j}^{\dot{\beta}}\right\} & =\delta_{j}^{i} \delta_{\dot{\alpha}}^{\dot{\beta}}(2 \mathbb{D}+Y)+4 \delta_{j}^{i} \bar{M}_{\dot{\alpha}}{ }^{\dot{\beta}}-4 \delta_{\dot{\alpha}}^{\dot{\beta}} J^{i}{ }_{j}, \\
{\left[K_{\alpha \dot{\alpha}}, \nabla_{\beta}^{j}\right] } & =-2 \mathrm{i} \varepsilon_{\alpha \beta} \bar{S}_{\dot{\alpha}}^{j}, \quad\left[K_{\alpha \dot{\alpha}}, \bar{\nabla}_{j}^{\dot{\beta}}\right]=-2 \mathrm{i} \delta_{\dot{\alpha}}^{\dot{\beta}} S_{\alpha j}, \\
{\left[S_{i}^{\alpha}, \nabla_{\beta \dot{\beta}}\right] } & =2 \mathrm{i} \delta_{\beta}^{\alpha} \bar{\nabla}_{\dot{\beta}}^{i}, \quad\left[\bar{S}_{\dot{\alpha}}^{i}, \nabla_{\beta \dot{\beta}}\right]=-2 \mathrm{i} \varepsilon_{\dot{\alpha} \dot{\beta}} \nabla_{\beta i},
\end{aligned}
$$

where all other graded commutators vanish. 
Consider a tensor superfield $\Psi$ with suppressed indices. It is said to be primary if

$$
K^{B} \Psi=0 .
$$

Additionally, its dimension $\Delta_{\Psi}$ and $\mathrm{U}(1)_{R}$ charge $q_{\Psi}$ are defined as follows:

$$
\mathbb{D} \Psi=\Delta_{\Psi} \Psi, \quad Y \Psi=q_{\Psi} \Psi .
$$

Of particular importance are (covariantly) chiral superfields, which satisfy

$$
\bar{\nabla}_{\dot{\alpha}}^{i} \Psi=0 \text {. }
$$

The consistency of this constraint with the superconformal algebra leads to highly nontrivial implications. In particular, it can carry no isospinor or dotted spinor indices, $\Psi=$ $\Psi_{\alpha(m)}$, and its $\mathrm{U}(1)_{R}$ charge and dimension are related as follows:

$$
q_{\Psi}=-2 \Delta_{\Psi} .
$$

Further, we note that for any primary tensor superfield $\Phi_{\alpha(m)}$ with the property $q_{\Phi}=$ $-2 \Delta_{\Phi}$, the following object

$$
\Psi_{\alpha(m)}=\bar{\nabla}^{4} \Phi_{\alpha(m)} \equiv \frac{1}{48} \bar{\nabla}^{i j} \bar{\nabla}_{i j} \Phi_{\alpha(m)}
$$

is both chiral and primary [40]. It is important to note that not all chiral primary superfields take this form, see e.g. (3.7).

In [19], it was shown that, in order to reproduce the component structure of conformal supergravity, certain constraints must be imposed on the graded commutators $\left[\nabla_{A}, \nabla_{B}\right\}$. In particular, they must be expressed solely in terms of the super-Weyl tensor, $W_{\alpha(2)}$, and its covariant derivatives. This superfield is primary, chiral and carries dimension 1,

$$
K^{B} W_{\alpha \beta}=0, \quad \bar{\nabla}_{\dot{\alpha}} W_{\alpha \beta}=0, \quad \mathbb{D} W_{\alpha \beta}=W_{\alpha \beta} .
$$

The solution to the aforementioned constraints are given by

$$
\begin{aligned}
\left\{\nabla_{\alpha}^{i}, \nabla_{\beta}^{j}\right\}= & 2 \varepsilon^{i j} \varepsilon_{\alpha \beta} \bar{W}_{\dot{\gamma} \dot{\delta}} \bar{M}^{\dot{\gamma} \dot{\delta}}+\frac{1}{2} \varepsilon^{i j} \varepsilon_{\alpha \beta} \bar{\nabla}_{\dot{\gamma} k} \bar{W}^{\dot{\gamma} \dot{\delta}} \bar{S}_{\dot{\delta}}^{k}-\frac{1}{2} \varepsilon^{i j} \varepsilon_{\alpha \beta} \nabla_{\gamma \dot{\delta}} \bar{W}_{\dot{\gamma}}^{\dot{\delta}} K^{\gamma \dot{\gamma}} \\
\left\{\nabla_{\alpha}^{i}, \bar{\nabla}_{j}^{\dot{\beta}}\right\}= & -2 \mathrm{i} \delta_{j}^{i} \nabla_{\alpha}^{\dot{\beta}} \\
{\left[\nabla_{\alpha \dot{\alpha}}, \nabla_{\beta}^{i}\right]=} & -\mathrm{i} \varepsilon_{\alpha \beta} \bar{W}_{\dot{\alpha} \dot{\beta}} \bar{\nabla}^{\dot{\beta} i}-\frac{\mathrm{i}}{2} \varepsilon_{\alpha \beta} \bar{\nabla}^{\dot{\beta} i} \bar{W}_{\dot{\alpha} \dot{\beta}} \mathbb{D}-\frac{\mathrm{i}}{4} \varepsilon_{\alpha \beta} \bar{\nabla}^{\dot{\beta} i} \bar{W}_{\dot{\alpha} \dot{\beta}} Y+\mathrm{i} \varepsilon_{\alpha \beta} \bar{\nabla}_{j}^{\dot{\beta}} \bar{W}_{\dot{\alpha} \dot{\beta}} J^{i j} \\
& -\mathrm{i} \varepsilon_{\alpha \beta} \bar{\nabla}_{\dot{\beta}}^{i} \bar{W}_{\dot{\gamma} \dot{\alpha}} \bar{M}^{\dot{\beta} \dot{\gamma}}-\frac{\mathrm{i}}{4} \varepsilon_{\alpha \beta} \bar{\nabla}_{\dot{\alpha}}^{i} \bar{\nabla}_{k}^{\dot{\beta}} \bar{W}_{\dot{\beta} \dot{\gamma}} \bar{S}^{\dot{\gamma} k}+\frac{1}{2} \varepsilon_{\alpha \beta} \nabla^{\gamma \dot{\beta}} \bar{W}_{\dot{\alpha} \dot{\beta}} S_{\gamma}^{i} \\
& +\frac{\mathrm{i}}{4} \varepsilon_{\alpha \beta} \bar{\nabla}_{\dot{\alpha}}^{i} \nabla_{\dot{\gamma}}^{\gamma} \bar{W}^{\dot{\gamma} \dot{\beta}} K_{\gamma \dot{\beta}} .
\end{aligned}
$$

We also find that $W_{\alpha \beta}$ obeys the Bianchi identity

$$
B=\nabla_{\alpha \beta} W^{\alpha \beta}=\bar{\nabla}^{\dot{\alpha} \dot{\beta}} \bar{W}_{\dot{\alpha} \dot{\beta}}=\bar{B},
$$

where $B$ is the $\mathcal{N}=2$ super-Bach tensor. The equation of motion for $\mathcal{N}=2$ conformal supergravity is the Bach-flatness condition

$$
\nabla_{\alpha \beta} W^{\alpha \beta}=\bar{\nabla}^{\dot{\alpha} \dot{\beta}} \bar{W}_{\dot{\alpha} \dot{\beta}}=0 .
$$

We point out that the $\mathcal{N}=1$ super-Bach tensor was introduced in [88], see also [3, 4, 84]. 
Open Access. This article is distributed under the terms of the Creative Commons Attribution License (CC-BY 4.0), which permits any use, distribution and reproduction in any medium, provided the original author(s) and source are credited.

\section{References}

[1] P.S. Howe, K.S. Stelle and P.K. Townsend, Supercurrents, Nucl. Phys. B 192 (1981) 332 [INSPIRE].

[2] E.S. Fradkin and A.A. Tseytlin, Conformal supergravity, Phys. Rept. 119 (1985) 233.

[3] S.M. Kuzenko, R. Manvelyan and S. Theisen, Off-shell superconformal higher spin multiplets in four dimensions, JHEP 07 (2017) 034 [arXiv: 1701.00682] [INSPIRE].

[4] S.M. Kuzenko and M. Ponds, Conformal geometry and (super)conformal higher-spin gauge theories, JHEP 05 (2019) 113 [arXiv: 1902.08010] [INSPIRE].

[5] S.M. Kuzenko and E.S.N. Raptakis, Symmetries of supergravity backgrounds and supersymmetric field theory, JHEP 04 (2020) 133 [arXiv: 1912.08552] [INSPIRE].

[6] S.M. Kuzenko, M. Ponds and E.S.N. Raptakis, New locally (super)conformal gauge models in Bach-flat backgrounds, JHEP 08 (2020) 068 [arXiv: 2005.08657] [INSPIRE].

[7] S.M. Kuzenko, M. Ponds and E.S.N. Raptakis, Generalised superconformal higher-spin multiplets, JHEP 03 (2021) 183 [arXiv:2011.11300] [INSPIRE].

[8] S.M. Kuzenko and D.X. Ogburn, Off-shell higher spin $N=2$ supermultiplets in three dimensions, Phys. Rev. D 94 (2016) 106010 [arXiv:1603.04668] [INSPIRE].

[9] S.M. Kuzenko, Higher spin super-Cotton tensors and generalisations of the linear-chiral duality in three dimensions, Phys. Lett. B 763 (2016) 308 [arXiv:1606.08624] [INSPIRE].

[10] S.M. Kuzenko and M. Tsulaia, Off-shell massive $N=1$ supermultiplets in three dimensions, Nucl. Phys. B 914 (2017) 160 [arXiv:1609.06910] [INSPIRE].

[11] S.M. Kuzenko and M. Ponds, Topologically massive higher spin gauge theories, JHEP 10 (2018) 160 [arXiv:1806.06643] [INSPIRE].

[12] J. Hutomo, S.M. Kuzenko and D. Ogburn, $\mathcal{N}=2$ supersymmetric higher spin gauge theories and current multiplets in three dimensions, Phys. Rev. D 98 (2018) 125004 [arXiv: 1807.09098] [INSPIRE].

[13] E.I. Buchbinder, D. Hutchings, J. Hutomo and S.M. Kuzenko, Linearised actions for $\mathcal{N}$ -extended (higher-spin) superconformal gravity, JHEP 08 (2019) 077 [arXiv:1905.12476] [INSPIRE].

[14] E.S. Fradkin and V.Y. Linetsky, A Superconformal Theory of Massless Higher Spin Fields in $D=(2+1)$, Annals Phys. 198 (1990) 293. [INSPIRE].

[15] E.S. Fradkin and V.Y. Linetsky, Superconformal Higher Spin Theory in the Cubic Approximation, Nucl. Phys. B 350 (1991) 274 [InSPIRE].

[16] E.S. Fradkin and M.A. Vasiliev, On the Gravitational Interaction of Massless Higher Spin Fields, Phys. Lett. B 189 (1987) 89 [InSPIRE].

[17] E.S. Fradkin and M.A. Vasiliev, Cubic Interaction in Extended Theories of Massless Higher Spin Fields, Nucl. Phys. B 291 (1987) 141 [INSPIRE]. 
[18] M.A. Vasiliev, Consistent equation for interacting gauge fields of all spins in (3+1)-dimensions, Phys. Lett. B 243 (1990) 378 [INSPIRE].

[19] D. Butter, $N=2$ Conformal Superspace in Four Dimensions, JHEP 10 (2011) 030 [arXiv: 1103.5914] [INSPIRE].

[20] V. Ogievetsky and E. Sokatchev, On Vector Superfield Generated by Supercurrent, Nucl. Phys. B 124 (1977) 309 [INSPIRE].

[21] E. Bergshoeff, M. de Roo and B. de Wit, Extended Conformal Supergravity, Nucl. Phys. B 182 (1981) 173 [INSPIRE].

[22] P.S. Howe, Supergravity in Superspace, Nucl. Phys. B 199 (1982) 309 [InSPIRE].

[23] S.M. Kuzenko, U. Lindström, M. Roček and G. Tartaglino-Mazzucchelli, $4 D N=2$ Supergravity and Projective Superspace, JHEP 09 (2008) 051 [arXiv:0805.4683] [INSPIRE].

[24] S.M. Kuzenko, U. Lindström, M. Roček and G. Tartaglino-Mazzucchelli, On conformal supergravity and projective superspace, JHEP 08 (2009) 023 [arXiv:0905.0063] [INSPIRE].

[25] B. de Wit, J.W. van Holten and A. Van Proeyen, Transformation Rules of $N=2$ Supergravity Multiplets, Nucl. Phys. B 167 (1980) 186 [InSPIRE].

[26] M. de Roo, J.W. van Holten, B. de Wit and A. Van Proeyen, Chiral Superfields in $N=2$ Supergravity, Nucl. Phys. B 173 (1980) 175 [inSPIRE].

[27] P.S. Howe and U. Lindström, Superconformal geometries and local twistors, JHEP 04 (2021) 140 [arXiv: 2012.03282] [INSPIRE].

[28] M.F. Sohnius, The Multiplet of Currents for $N=2$ Extended Supersymmetry, Phys. Lett. B 81 (1979) 8 [INSPIRE].

[29] S.M. Kuzenko and S. Theisen, Correlation functions of conserved currents in $N=2$ superconformal theory, Class. Quant. Grav. 17 (2000) 665 [hep-th/9907107] [INSPIRE].

[30] P.S. Howe and U. Lindström, Notes on Super Killing Tensors, JHEP 03 (2016) 078 [arXiv:1511.04575] [INSPIRE].

[31] P.S. Howe and U. Lindström, Some remarks on (super)-conformal Killing-Yano tensors, JHEP 11 (2018) 049 [arXiv: 1808.00583] [INSPIRE].

[32] S.M. Kuzenko and E.S.N. Raptakis, work in progress.

[33] P.S. Howe and U. Lindström, Super-Laplacians and their symmetries, JHEP 05 (2017) 119 [arXiv: 1612.06787] [INSPIRE].

[34] D. Butter, $N=1$ Conformal Superspace in Four Dimensions, Annals Phys. 325 (2010) 1026 [arXiv:0906.4399] [INSPIRE].

[35] A. Ceresole, G. Dall'Agata, R. D'Auria and S. Ferrara, Spectrum of type IIB supergravity on $A d S_{5} \times T^{11}$ : Predictions on $N=1$ SCFT's, Phys. Rev. D 61 (2000) 066001 [hep-th/9905226] [INSPIRE].

[36] S. Ferrara and B. Zumino, Transformation Properties of the Supercurrent, Nucl. Phys. B 87 (1975) 207 [INSPIRE].

[37] S. Ferrara, J. Wess and B. Zumino, Supergauge Multiplets and Superfields, Phys. Lett. B 51 (1974) 239 [INSPIRE].

[38] W. Siegel and S.J. Gates Jr., Superprojectors, Nucl. Phys. B 189 (1981) 295 [inSPIRE]. 
[39] S.J. Gates, M.T. Grisaru, M. Roček and W. Siegel, Superspace Or One Thousand and One Lessons in Supersymmetry, vol. 58 of Frontiers in Physics (1983) [hep-th/0108200] [INSPIRE].

[40] S.M. Kuzenko and G. Tartaglino-Mazzucchelli, Different representations for the action principle in $4 D N=2$ supergravity, JHEP 04 (2009) 007 [arXiv:0812.3464] [INSPIRE].

[41] D. Butter and S.M. Kuzenko, $N=2$ supergravity and supercurrents, JHEP 12 (2010) 080 [arXiv: 1011.0339] [INSPIRE].

[42] S.M. Kuzenko and E.S.N. Raptakis, Duality-invariant superconformal higher-spin models, Phys. Rev. D 104 (2021) 125003 [arXiv:2107.02001] [INSPIRE].

[43] S.M. Kuzenko and M. Ponds, Generalised conformal higher-spin fields in curved backgrounds, JHEP 04 (2020) 021 [arXiv: 1912.00652] [INSPIRE].

[44] J. van Muiden and A. Van Proeyen, The $\mathcal{N}=3$ Weyl multiplet in four dimensions, JHEP 01 (2019) 167 [arXiv: 1702.06442] [INSPIRE].

[45] S. Hegde and B. Sahoo, Comment on "The $N=3$ Weyl multiplet in four dimensions", Phys. Lett. B 791 (2019) 92 [arXiv:1810.05089] [INSPIRE].

[46] S. Hegde, M. Mishra and B. Sahoo, $N=3$ Conformal Supergravity in Four Dimensions, arXiv : 2104.07453 [INSPIRE].

[47] D. Butter, F. Ciceri, B. de Wit and B. Sahoo, Construction of all $N=4$ conformal supergravities, Phys. Rev. Lett. 118 (2017) 081602 [arXiv:1609.09083] [INSPIRE].

[48] D. Butter, F. Ciceri and B. Sahoo, $N=4$ conformal supergravity: the complete actions, JHEP 01 (2020) 029 [arXiv: 1910.11874] [INSPIRE].

[49] D. Butter, S.M. Kuzenko, J. Novak and G. Tartaglino-Mazzucchelli, Conformal supergravity in three dimensions: New off-shell formulation, JHEP 09 (2013) 072 [arXiv: 1305.3132] [INSPIRE].

[50] S. Ferrara, M. Kaku, P.K. Townsend and P. van Nieuwenhuizen, Gauging the Graded Conformal Group with Unitary Internal Symmetries, Nucl. Phys. B 129 (1977) 125 [INSPIRE].

[51] J.-H. Park, Superconformal symmetry and correlation functions, Nucl. Phys. B 559 (1999) 455 [hep-th/9903230] [INSPIRE].

[52] T. Nutma and M. Taronna, On conformal higher spin wave operators, JHEP 06 (2014) 066 [arXiv: 1404.7452] [INSPIRE].

[53] M. Grigoriev and A.A. Tseytlin, On conformal higher spins in curved background, J. Phys. A 50 (2017) 125401 [arXiv: 1609.09381] [INSPIRE].

[54] M. Beccaria and A.A. Tseytlin, On induced action for conformal higher spins in curved background, Nucl. Phys. B 919 (2017) 359 [arXiv:1702.00222] [INSPIRE].

[55] R. Manvelyan and G. Poghosyan, Geometrical structure of Weyl invariants for spin three gauge field in general gravitational background in $d=4$, Nucl. Phys. B 937 (2018) 1 [arXiv: 1804.10779] [INSPIRE].

[56] M.A. Vasiliev, Consistent Equations for Interacting Massless Fields of All Spins in the First Order in Curvatures, Annals Phys. 190 (1989) 59 [INSPIRE].

[57] N.G. Misuna and M.A. Vasiliev, Off-Shell Scalar Supermultiplet in the Unfolded Dynamics Approach, JHEP 05 (2014) 140 [arXiv:1301.2230] [INSPIRE]. 
[58] O.A. Gelfond and M.A. Vasiliev, Higher-Rank Fields and Currents, JHEP 10 (2016) 067 [arXiv: 1312.6673] [INSPIRE].

[59] I.A. Bandos, E. Ivanov, J. Lukierski and D. Sorokin, On the superconformal flatness of AdS superspaces, JHEP 06 (2002) 040 [hep-th/0205104] [INSPIRE].

[60] S.M. Kuzenko and G. Tartaglino-Mazzucchelli, Field theory in $4 D N=2$ conformally flat superspace, JHEP 10 (2008) 001 [arXiv:0807.3368] [INSPIRE].

[61] A.A. Tseytlin, On limits of superstring in $A d S_{5} \times S^{5}$, Theor. Math. Phys. 133 (2002) 1376 [hep-th/0201112] [INSPIRE].

[62] A.Y. Segal, Conformal higher spin theory, Nucl. Phys. B 664 (2003) 59 [hep-th/0207212] [INSPIRE].

[63] X. Bekaert, E. Joung and J. Mourad, Effective action in a higher-spin background, JHEP 02 (2011) 048 [arXiv: 1012.2103] [InSPIRE].

[64] R. Bonezzi, Induced Action for Conformal Higher Spins from Worldline Path Integrals, Universe 3 (2017) 64 [arXiv:1709.00850] [INSPIRE].

[65] A. Galperin, E. Ivanov, S. Kalitsyn, V. Ogievetsky and E. Sokatchev, Unconstrained $N=2$ Matter, Yang-Mills and Supergravity Theories in Harmonic Superspace, Class. Quant. Grav. 1 (1984) 469 [Erratum ibid. 2 (1985) 127] [INSPIRE].

[66] A.S. Galperin, E.A. Ivanov, V.I. Ogievetsky and E.S. Sokatchev, Harmonic Superspace, Cambridge University Press, Cambridge U.K. (2001) [DOI].

[67] A. Karlhede, U. Lindström and M. Roček, Selfinteracting Tensor Multiplets in $N=2$ Superspace, Phys. Lett. B 147 (1984) 297 [InSPIRE].

[68] U. Lindström and M. Roček, New HyperKähler Metrics and New Supermultiplets, Commun. Math. Phys. 115 (1988) 21 [inSPIRE].

[69] U. Lindström and M. Roček, $N=2$ SuperYang-Mills Theory in Projective Superspace, Commun. Math. Phys. 128 (1990) 191 [InSPIRE].

[70] S.M. Kuzenko, Lectures on nonlinear sigma-models in projective superspace, J. Phys. A 43 (2010) 443001 [arXiv: 1004.0880] [INSPIRE].

[71] R. Grimm, M. Sohnius and J. Wess, Extended Supersymmetry and Gauge Theories, Nucl. Phys. B 133 (1978) 275 [InSPIRE].

[72] L. Mezincescu, On the superfield formulation of O(2) supersymmetry, Dubna preprint JINR-P2-12572 (1979).

[73] D. Butter and S.M. Kuzenko, New higher-derivative couplings in $4 D N=2$ supergravity, JHEP 03 (2011) 047 [arXiv: 1012.5153] [INSPIRE].

[74] P. Breitenlohner and M.F. Sohnius, Superfields, Auxiliary Fields, and Tensor Calculus for $N=2$ Extended Supergravity, Nucl. Phys. B 165 (1980) 483 [INSPIRE].

[75] P. Breitenlohner and M.F. Sohnius, An Almost Simple Off-shell Version of SU(2) Poincaré Supergravity, Nucl. Phys. B 178 (1981) 151 [inSPIRE].

[76] M.F. Sohnius, K.S. Stelle and P.C. West, Representations of extended supersymmetry, in Superspace and Supergravity, S.W. Hawking and M. Roček (Eds.), Cambridge University Press, Cambridge, (1981) 283 [DOI]. 
[77] C. Fronsdal, Singletons and Massless, Integral Spin Fields on de Sitter Space (Elementary Particles in a Curved Space. 7, Phys. Rev. D 20 (1979) 848 [InSPIRE].

[78] J. Fang and C. Fronsdal, Massless, Half Integer Spin Fields in de Sitter Space, Phys. Rev. D 22 (1980) 1361 [INSPIRE].

[79] S.M. Kuzenko and A.G. Sibiryakov, Free massless higher superspin superfields on the anti-de Sitter superspace, Phys. Atom. Nucl. 57 (1994) 1257 [arXiv:1112.4612] [INSPIRE].

[80] E.I. Buchbinder, J. Hutomo and S.M. Kuzenko, Higher spin supercurrents in anti-de Sitter space, JHEP 09 (2018) 027 [arXiv: 1805. 08055] [INSPIRE].

[81] S.J. Gates Jr., S.M. Kuzenko and A.G. Sibiryakov, $N=2$ supersymmetry of higher superspin massless theories, Phys. Lett. B 412 (1997) 59 [hep-th/9609141] [InSPIRE].

[82] S.J. Gates Jr., S.M. Kuzenko and A.G. Sibiryakov, Towards a unified theory of massless superfields of all superspins, Phys. Lett. B 394 (1997) 343 [hep-th/9611193] [INSPIRE].

[83] D. Butter and S.M. Kuzenko, $N=2$ AdS supergravity and supercurrents, JHEP 07 (2011) 081 [arXiv: 1104.2153] [INSPIRE].

[84] I.L. Buchbinder and S.M. Kuzenko, Ideas and Methods of Supersymmetry and Supergravity or a Walk Through Superspace, Revised Edition, IOP, Bristol, (1998) [ISBN: 9780750305068].

[85] E.I. Buchbinder, D. Hutchings, S.M. Kuzenko and M. Ponds, AdS superprojectors, JHEP 04 (2021) 074 [arXiv:2101.05524] [INSPIRE].

[86] D. Butter and J. Novak, Component reduction in $N=2$ supergravity: the vector, tensor, and vector-tensor multiplets, JHEP 05 (2012) 115 [arXiv:1201.5431] [INSPIRE].

[87] J. Wess and J. Bagger, Supersymmetry and Supergravity, Second Edition, Princeton University Press, Princeton (1992) [ISBN: 9780691025308].

[88] I.L. Buchbinder and S.M. Kuzenko, Quantization of the classically equivalent theories in the superspace of simple supergravity and quantum equivalence, Nucl. Phys. B 308 (1988) 162 [INSPIRE]. 